\title{
Passivity Analysis for Non-Linear, Non-Stationary Entry Capsules: Rotational Motion
}

\author{
E. Mooij * \\ Delft University of Technology, Faculty of Aerospace Engineering, \\ Kluyverweg 1, 2629 HS Delft, The Netherlands
}

\begin{abstract}
To analyze the passivity of non-linear, time-varying systems we study an entry capsule that enters the atmosphere in a lift-down configuration (i.e., a bank angle larger than $90^{\circ}$ ) to avoid skipping flight, and which is controlled by a Reaction Control System only. Deriving the passivity conditions for rotational motion, and evaluating these conditions along the trajectory shows that the (non-linear) entry capsule is Almost Strictly Passive, and the sufficient conditions to apply Simple Adaptive Control are met. Further, the preliminary design of an attitude-control system using the theory of Simple Adaptive Control is discussed and analyzed. The two designs for both a low and a high dynamic-pressure operating condition show an excellent performance. Each design uses its own controller parameters (i.e., weighting matrices, zero integral gain and integral-gain filter parameter), which necessitates the use of an interpolation scheme once a trajectory with changing operating conditions is flown. The nominal mission (entry at $220 \mathrm{~km}$ altitude with a velocity of $11 \mathrm{~km} / \mathrm{s}$, and final conditions at Mach = 1) can be flown without any difficulty. The angle-of-attack command is provided by a trim law, and although some deviations are observed when the capsule becomes less aerodynamically stable, this deviation does not diverge in spite of the pitch-thruster saturation. Responses due to errors in the initial attitude and angular rates show smooth curves in both attitude motion and thruster controls. A Monte-Carlo analysis that includes errors in the initial attitude, angular rate and inertia properties shows similar results, and it can be concluded that the performance of the controller given the current dispersions is satisfying.
\end{abstract}

\section{Introduction}

Previous publications have presented successful application of Simple Adaptive Control (SAC) in the field of autopilot design, ${ }^{1,2}$ robotics ${ }^{3}{ }^{\text {optical telescopes }}{ }^{4}$ and re-entry vehicles. ${ }^{5,6}$ However, as adaptive controllers (as well as fuzzy controllers and neuro-controllers) use non-stationary control, one must have a guarantee of stability that goes beyond the cases that are presented in simulations. Otherwise, even slight changes in the real-world environment may result in grave deterioration of performance and may even lead to total instability. The main problem with non-stationary systems or controllers is that the proofs of stability could be very difficult, in particular for larger systems. Therefore, one may try to replace the theoretical proofs with practical tests and demonstrations of stable behavior. One may feel sufficiently confident to believing that a system is stable after one had tested it for almost all imaginable conditions. On the other hand, it may be quite difficult to find that specific situation that may destabilize the non-stationary system.

Such examples, along with past experience, may explain why in adaptive control one takes the pain of attempting to find rigorous proofs of stability and clarify the passivity conditions that are needed to find the proofs. It is the designer decision whether to use the methodology (i.e., adaptive, fuzzy, neuro-controller, etc.) that works, and just test it in various environmental conditions, or take the pain of going through the thorough theoretical analysis. To apply Simple Adaptive Control theory to non-linear and non-stationary systems, it needs to be Almost Strictly Passive (ASP), which is an extension of the Almost Strictly Positive Real (ASPR) condition for linear, time-invariant systems. ${ }^{2}$ To fulfill the ASP condition, the controlled, nonlinear system has to be minimum-phase (i.e., it needs to have stable zero dynamics), and there is a specific condition for the product of output and input matrix, which will be detailed later on in this paper.

\footnotetext{
*Assistant Professor, section Astrodynamics and Space Missions, e.mooij@tudelft.nl, Senior Member AIAA.
} 
The use of SAC for the longitudinal and lateral control of winged re-entry vehicles has resulted in very robust controllers that remain stable in the presence of vehicular and environmental uncertainties, even when some of the actuators are saturated for a noticeable period of time. ${ }^{6}$ However, it was actually never analyzed why the performance of SAC was so good, as it was applied to the non-linear and non-stationary vehicle $a d$ $h o c$, i.e., it was not checked whether the system is ASP or not.

From previous work ${ }^{8}$ it was found that the linearized system was, in fact, not ASPR, which was not an encouraging start for analyzing the non-linear system, despite the very good performance of the actual guidance and control system. The straightforward approach of solving the differential ASP equations for the general non-linear case appeared to be a very big, if not impossible, task, and we concluded that the system under study had to be either simplified or changed. Therefore, to continue this work we take one step back. Two issues prevented us from proving that even the LTI system was ASPR, the first being the fact that the system was non-minimum phase when flying with zero bank angle. The second issue basically showed that whenever there is hybrid control, i.e., multiple control actuators for a single axis (i.e., yaw control was established by combined reaction and aerodynamic control), the second ASPR condition cannot be met.

Therefore, we will be proving the passivity for a slightly less demanding system, i.e., an Apollo-shaped re-entry capsule that enters the atmosphere in a lift-down configuration (i.e., a bank angle larger than $90^{\circ}$ ) and has only reaction-control thrusters as control actuators. Without going into detail of the actual GNC system design, for the sake of the discussion we assume that both guidance and control system will be based on Simple Adaptive Control theory. Since the translational motion is discussed elsewhere, ${ }^{12}$ here we will focus on the rotational motion and thus on the control system that has to enforce closed-loop stability throughout the flight.

The layout of this paper is as follows. Section II will give some background material on Simple Adaptive Control theory and stability analysis of non-linear systems. In Section III, the model is described as well as a summary of the flight mechanics of the entry capsule. Section IV presents the results of the passivity analysis. A typical example of Simple Adaptive Control is discussed in Section V, where the preliminary control-system design of the entry capsule is analyzed. Section VI, finally, concludes this paper.

\section{Theoretical Background}

\section{A. Simple Adaptive Control}

In this section, some background material will be presented on the set-up of an MRAG and/or MRAC system, taken from a recent work on direct adaptive control algorithms. ${ }^{2}$ A favorable approach is that of Simple Adaptive Control based on output feedback. The algorithm aims at matching the response of the system that is to be controlled (the plant) to that of a reference model (the model). The basic adaptive algorithm to compute the plant input $\mathbf{u}_{\mathbf{p}}$ is as follows (Fig. 1):

$$
\mathbf{u}_{\mathbf{p}}(t)=\mathbf{K}_{\mathbf{r}}(t) \mathbf{r}(t)
$$

where

$$
\mathbf{r}(t)=\left[\mathbf{e}_{\mathbf{y}}(t) \mathbf{x}_{\mathbf{m}}(t) \mathbf{u}_{\mathbf{m}}\right]^{T}
$$

and

$$
\mathbf{K}_{\mathbf{r}}(t)=\left[\begin{array}{lll}
\mathbf{K}_{\mathbf{e}}(t) & \mathbf{K}_{\mathbf{x}}(t) & \mathbf{K}_{\mathbf{u}}(t)
\end{array}\right]
$$

To compute the adaptive gains, $\mathbf{K}_{\mathbf{r}}$ is defined to be the sum of an integral and a proportional component, i.e.,

$$
\mathbf{K}_{r}(t)=\mathbf{K}_{i}(t)+\mathbf{K}_{p}(t)
$$

with

$$
\begin{gathered}
\dot{\mathbf{K}}_{\mathbf{i}}(t)=\mathbf{e}_{\mathbf{y}} \mathbf{r}^{T}(t) \mathbf{T}_{\mathbf{i}} \\
\mathbf{K}_{\mathbf{p}}(t)=\mathbf{e}_{\mathbf{y}}(t) \mathbf{r}^{T}(t) \mathbf{T}_{\mathbf{p}}
\end{gathered}
$$




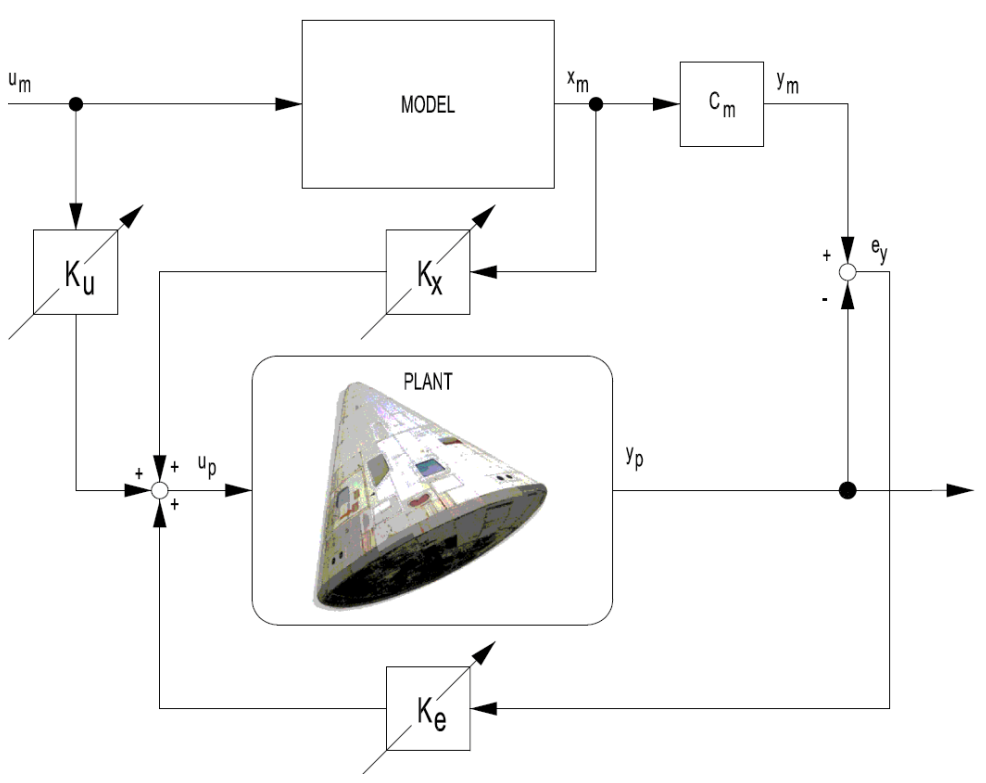

Figure 1. Basic architecture of a Simple Adaptive Control algorithm.

It can be seen that the model input $\mathbf{u}_{\mathbf{m}}$ and model state $\mathbf{x}_{\mathbf{m}}$ are required to form part of the input signal $\mathbf{u}_{\mathbf{p}}$ to the plant. Moreover, the so-called output error $\mathbf{e}_{\mathbf{y}}$ serves as a feedback quantity to form the third element that composes $\mathbf{u}_{\mathbf{p}}$. The three gains, i.e., $\mathbf{K}_{\mathbf{x}}, \mathbf{K}_{\mathbf{u}}$ and $\mathbf{K}_{\mathbf{e}}$, are adaptive. The weighting matrices $\mathbf{T}_{\mathbf{p}}$ and $\mathbf{T}_{\mathbf{i}}$ are positive semi-definite and positive definite, respectively. Note that the proportional gain component has a direct influence on the transient tracking behavior, but is strictly speaking not required to enforce asymptotic tracking. This is guaranteed by the integral gain. To improve the transient response by only using an integral gain, a constant gain value can be added to $\mathbf{K}_{\mathbf{i}}$. An advantage over the use of the proportional gain is that this constant value is independent of $\mathbf{e}_{\mathbf{y}}$, and is therefore non-zero even if $\mathbf{e}_{\mathbf{y}}$ is zero. In that case, the integral gain derived from Eq. (6) becomes

$$
\mathbf{K}_{\mathbf{i}}(t)=\mathbf{K}_{\mathbf{i}, \mathbf{0}}+\int_{0}^{t} \dot{\mathbf{K}}_{\mathbf{i}}(t) d t
$$

The damping of the system can be improved by including the error derivatives in the output error vector. Thus, the error for output $\mathbf{y}$ becomes:

$$
\mathbf{e}_{\mathbf{y}}=\mathbf{K}_{\mathbf{y}}^{\mathbf{T}}\left(\mathbf{y}_{\mathbf{m}}-\mathbf{y}_{\mathbf{p}}\right)+\mathbf{K}_{\dot{\mathbf{y}}}^{\mathbf{T}}\left(\dot{\mathbf{y}}_{\mathbf{m}}-\dot{\mathbf{y}}_{\mathbf{p}}\right)
$$

with $\mathbf{K}_{\mathbf{y}}^{\mathbf{T}}$ and $\mathbf{K}_{\dot{\mathbf{y}}}^{\mathbf{T}}$ being a proportional and derivative output gain, respectively.

To guarantee that all states and gains in the adaptive system are bounded and the output error is asymptotically stable, it is necessary that a linear plant is ASPR. This means, for practical purposes, that the plant can be stabilized by any sufficiently high, constant or time-variable, output gain. For time-varying, non-linear plants there is the similar condition of ASP. Note that it is not trivial whether a non-linear system is ASP or not, so in some cases the controller is designed ad hoc and validated by extensive simulation. To apply the adaptive algorithm to a much wider class of systems, various modifications have been developed. One modification is to augment the plant with a so-called feed-forward compensator, such that the class of ASPR systems is increased. If the compensator dynamics is given by the strictly proper transfer function matrix $\mathbf{G}_{\mathbf{c}}(s)$ with realization $\dot{\mathbf{x}}_{\mathbf{c}}=\mathbf{A}_{\mathbf{c}} \mathbf{x}_{\mathbf{c}}(t)+\mathbf{B}_{\mathbf{c}} \mathbf{u}_{\mathbf{p}}(t)$ and $\mathbf{y}_{\mathbf{c}}=\mathbf{C}_{\mathbf{c}} \mathbf{x}_{\mathbf{c}}(t)$ such that the augmented output to be controlled is $\mathbf{y}_{\mathbf{a}}(t)=\mathbf{y}_{\mathbf{p}}(t)+\mathbf{y}_{\mathbf{c}}(t)$ then the augmented system $\mathbf{G}_{\mathbf{a}}(s)=\mathbf{G}_{\mathbf{p}}(s)+\mathbf{G}_{\mathbf{c}}(s)$ is ASPR provided that:

- $\mathbf{G}_{\mathbf{c}}(s)$ is such that the relative degree of $\mathbf{G}_{\mathbf{a}}(s)$ is $m$ (number of inputs), and 
- $\mathbf{G}_{\mathbf{c}}^{-1}$ stabilizes the closed-loop output feedback system.

In general, while using plant feed-forward compensators the model-following error will be bounded but not zero. This can be alleviated by a second extension to the adaptive algorithm, i.e., to apply not only feed-forward around the plant but also around the reference model.

So far, an ideal environment has been considered. To cope with environmental disturbances that lead to a persistent non-zero error and therefore to a continuous change in the integral gain $\mathbf{K}_{\mathbf{i}}$, a robust design can be applied to adjust the integral gain and preventing it from reaching very high values. The integral term of Eq. (6) is adjusted as follows:

$$
\dot{\mathbf{K}}_{\mathbf{i}}=\mathbf{e}_{\mathbf{y}}(t) \mathbf{r}^{T} \mathbf{T}_{\mathbf{i}}-\sigma_{i} \mathbf{K}_{\mathbf{i}}(t)
$$

Without the $\sigma_{i}$-term, $\mathbf{K}_{\mathbf{i}}(t)$ is a perfect integrator and may steadily increase (and even diverge) whenever perfect output following is not possible. Including the $\sigma_{i}$-term, $\mathbf{K}_{\mathbf{i}}(t)$ is obtained from a first-order filtering of $\mathbf{e}_{\mathbf{y}}(t) \mathbf{r}^{\mathbf{T}} \mathbf{T}_{\mathbf{i}}$ and, therefore, cannot diverge, unless the output error diverges.

\section{B. Sufficient Conditions}

Suppose that the plant to be controlled is LTI and given in state-space form by

$$
\begin{aligned}
& \dot{\mathbf{x}}_{\mathbf{p}}=\mathbf{A}_{\mathbf{p}} \mathbf{x}_{\mathbf{p}}(t)+\mathbf{B}_{\mathbf{p}} \mathbf{u}_{\mathbf{p}}(t) \\
& \mathbf{y}_{\mathbf{p}}=\mathbf{C}_{\mathbf{p}} \mathbf{x}_{\mathbf{p}}(t)
\end{aligned}
$$

It can be shown that any strictly minimum-phase LTI system with a positive definite symmetric product $\mathbf{C}_{\mathbf{p}} \mathbf{B}_{\mathbf{p}}$ is ASPR. In other words, considering a (fictitious) constant output feedback controller, i.e., $\mathbf{u}_{\mathbf{p}}=$

$-\mathbf{K}_{\mathbf{e}} \mathbf{y}_{\mathbf{p}}$, there exist two positive definite symmetric matrices $\mathbf{P}$ and $\mathbf{Q}$ such that the fictitious closed-loop system using the unknown gain $\mathbf{K}_{\mathbf{e}}$ satisfies simultaneously the following SPR conditions:

$$
\begin{gathered}
\mathbf{P}\left[\mathbf{A}_{\mathbf{p}}-\mathbf{B}_{\mathbf{p}} \mathbf{K}_{\mathbf{e}} \mathbf{C}_{\mathbf{p}}\right]+\left[\mathbf{A}_{\mathbf{p}}-\mathbf{B}_{\mathbf{p}} \mathbf{K}_{\mathbf{e}} \mathbf{C}_{\mathbf{p}}\right]^{T} \mathbf{P}= \\
\mathbf{P A}_{\mathbf{K}}+\mathbf{A}_{\mathbf{K}}^{\mathbf{T}} \mathbf{P}=-\mathbf{Q}<\mathbf{0} \\
\mathbf{P B}_{\mathbf{p}}=\mathbf{C}_{\mathbf{p}}^{\mathbf{T}}
\end{gathered}
$$

Thus, the original plant is denoted as ASPR, because only a constant feedback gain separates it from being SPR.

Eq. (11) shows that the fictitious closed-loop SPR system is asymptotically stable, whereas from Eq. (12) one gets the condition $\mathbf{C}_{\mathbf{p}} \mathbf{B}_{\mathbf{p}}=\mathbf{B}_{\mathbf{p}}^{\mathbf{T}} \mathbf{C}_{\mathbf{p}}^{\mathbf{T}}=\mathbf{B}_{\mathbf{p}}^{\mathbf{T}} \mathbf{P} \mathbf{B}_{\mathbf{p}}>\mathbf{0}$ (i.e., positive definite symmetric). Using the ASPR conditions and setting up a Lyapunov equation for the adaptive closed-loop system, one can prove that the adaptive system is stable. It is worth mentioning that only the transfer function should rigorously be called SPR or ASPR, while the system itself should be called Strictly Passive or Almost Strictly Passive. An important extension led to the applicability of the important SPR and ASPR properties to the class of systems where the non-symmetric $\mathbf{C}_{\mathbf{p}} \mathbf{B}_{\mathbf{p}}$ is diagonizable and has real positive eigenvalues. ${ }^{9}$

These concepts can be extended to non-linear time-varying systems, and similar (though more complicated) expressions can be derived. ${ }^{3}$ Let the non-stationary plant to be controlled be given in state-space form by

$$
\begin{aligned}
& \dot{\mathbf{x}}_{\mathbf{p}}=\mathbf{A}_{\mathbf{p}}\left(\mathbf{x}_{\mathbf{p}}, t\right) \mathbf{x}_{\mathbf{p}}(t)+\mathbf{B}_{\mathbf{p}}\left(\mathbf{x}_{\mathbf{p}}, t\right) \mathbf{u}_{\mathbf{p}}(t) \\
& \mathbf{y}_{\mathbf{p}}=\mathbf{C}_{\mathbf{p}}\left(\mathbf{x}_{\mathbf{p}}, t\right) \mathbf{x}_{\mathbf{p}}(t)
\end{aligned}
$$

The need for the ASP condition appears during the proof of stability of the adaptive control system using Lyapunov functions of the form

$$
\begin{aligned}
V(t) & =\mathbf{e}_{\mathbf{x}}^{\mathbf{T}}(t) \mathbf{P} \mathbf{e}_{\mathbf{x}}(t)+ \\
& +\operatorname{trace}\left\{\mathbf{S}[\mathbf{K}(t)-\mathbf{K}] \mathbf{T}_{\mathbf{i}}^{-\mathbf{1}}[\mathbf{K}(t)-\mathbf{K}]^{T} \mathbf{S}^{\mathbf{T}}\right\}
\end{aligned}
$$


In Eq. (14) $\mathbf{K}$ is an (unknown) ideal constant gain that would allow asymptotically perfect following. The Lyapunov function contains the constant nonsingular matrix $\mathbf{S}$ that allows mitigating the symmetry condition on $\mathbf{C}_{\mathbf{p}} \mathbf{B}_{\mathbf{p}}$. Defining $\mathbf{W}=\mathbf{S}^{\mathbf{T}} \mathbf{S}$, one can show that stability of the nonlinear system with adaptive control is guaranteed if there exist two uniformly positive definite symmetric matrices, $\mathbf{P}\left(\mathbf{x}_{\mathbf{p}}, t\right)$ and $\mathbf{Q}\left(\mathbf{x}_{\mathbf{p}}, t\right)$, and the constant positive definite symmetric matrix $\mathbf{W}$ such that the following ASP conditions are satisfied:

$$
\begin{gathered}
\dot{\mathbf{P}}+\mathbf{P}\left[\mathbf{A}_{\mathbf{p}}-\mathbf{B}_{\mathbf{p}} \mathbf{K}_{\mathbf{e}} \mathbf{C}_{\mathbf{p}}\right]+\left[\mathbf{A}_{\mathbf{p}}-\mathbf{B}_{\mathbf{p}} \mathbf{K}_{\mathbf{e}} \mathbf{C}_{\mathbf{p}}\right]^{T} \mathbf{P} \\
=\mathbf{P A}_{\mathbf{K}}+\mathbf{A}_{\mathbf{K}}^{\mathbf{T}} \mathbf{P}=-\mathbf{Q}<\mathbf{0} \\
\mathbf{P B}_{\mathbf{p}}=\mathbf{C}_{\mathbf{p}}^{\mathbf{T}} \mathbf{W}
\end{gathered}
$$

From (16) one gets

$$
\mathbf{B}_{\mathrm{p}}^{\mathrm{T}} \mathbf{P} \mathbf{B}_{\mathrm{p}}=\mathbf{B}_{\mathrm{p}}^{\mathrm{T}} \mathbf{C}_{\mathbf{p}}^{\mathrm{T}} \mathbf{W}=\mathbf{W C}_{\mathbf{p}} \mathbf{B}_{\mathbf{p}}
$$

Although conditions (15)-(17) may still look obscure for the designer, it has been shown that they are satisfied for any non-stationary system that satisfies the following two conditions:

1. The controlled plant is minimum-phase (i.e., it has stable zero dynamics), and

2. The not-necessarily symmetric positive definite product $\mathbf{C}_{\mathbf{p}} \mathbf{B}_{\mathbf{p}}$ can be made symmetric via a constant transformation $\mathbf{S}$, namely:

$$
\mathbf{C}_{\mathbf{p}}\left(\mathbf{x}_{\mathbf{p}}, t\right) \mathbf{B}_{\mathbf{p}}\left(\mathbf{x}_{\mathbf{p}}, t\right) \mathbf{S}=\mathbf{S R}\left(\mathbf{x}_{\mathbf{p}}, t\right)
$$

Here, $\mathbf{R}\left(\mathbf{x}_{\mathbf{p}}, t\right)$ is symmetric.

While the test of condition (18) is simple and is trivially satisfied when $\mathbf{C}_{\mathbf{p}} \mathbf{B}_{\mathbf{p}}$ is symmetric, the test of zero-dynamics stability is more complex in non-stationary systems. To this end, one defines two matrices, $\mathbf{N}\left(\mathbf{x}_{\mathbf{p}}, t\right)$ and $\mathbf{M}\left(\mathbf{x}_{\mathbf{p}}, t\right)$, that satisfy the relations

$$
\mathbf{C}\left(\mathbf{x}_{\mathbf{p}}, t\right) \mathbf{M}\left(\mathbf{x}_{\mathbf{p}}, t\right)=\mathbf{0} \quad \mathbf{N}\left(\mathbf{x}_{\mathbf{p}}, t\right) \mathbf{B}\left(\mathbf{x}_{\mathbf{p}}, t\right)=\mathbf{0} \quad \mathbf{N}\left(\mathbf{x}_{\mathbf{p}}, t\right) \mathbf{M}\left(\mathbf{x}_{\mathbf{p}}, t\right)=\mathbf{I}
$$

These matrices always exist, and one can use the transformation

$$
\mathbf{x}_{\mathbf{p}}=\mathbf{M}\left(\mathbf{x}_{\mathbf{p}}, t\right) \mathbf{z}
$$

which leads to the differential equation

$$
\dot{\mathbf{z}}(t)=\left(\dot{\mathbf{N}}\left(\mathbf{x}_{\mathbf{p}}, t\right)+\mathbf{N}\left(\mathbf{x}_{\mathbf{p}}, t\right) \mathbf{A}_{\mathbf{p}}\left(\mathbf{x}_{\mathbf{p}}, t\right)\right) \mathbf{M}\left(\mathbf{x}_{\mathbf{p}}, t\right) \mathbf{z}(t)
$$

This is called the zero dynamics because it isolates the output from the input, as the output that corresponds to this dynamics is

$$
\mathbf{y}_{\mathbf{p}}(t)=\mathbf{C}_{\mathbf{p}}\left(\mathbf{x}_{\mathbf{p}}, t\right) \mathbf{x}_{\mathbf{p}}=\mathbf{C}_{\mathbf{p}}\left(\mathbf{x}_{\mathbf{p}}, t\right) \mathbf{M}\left(\mathbf{x}_{\mathbf{p}}, t\right) \mathbf{z}(t)=\mathbf{0}
$$

Therefore, one must find appropriate matrices $\mathbf{N}$ and $\mathbf{M}$ and test condition (18) and stability of the zero-dynamics (21). If condition (18) is not too difficult to check and satisfy, checking Eq. (21) seems to be a rather elaborate task for a general non-stationary non-linear system, although it becomes quite simple in special classes of systems, such as robotic manipulators, etc. ${ }^{3}$ In those nonlinear systems where the parameters may change from case to case, yet they can be considered constant during the task, it is sufficient to treat the system as LTI and check conditions in extreme situations. ${ }^{1,3}$ From previous work, ${ }^{6}$ it has become clear that the that the transient effect (i.e., time-varying aspects) plays less of a role while studying the rotational motion. Therefore, for the stability analysis we can limit ourselves to scaled-down intervals of the total flight regime and assume the translational motion to be in equilibrium. 


\section{Model Description}

The model that is used in this study is that of an Apollo-like entry capsule (mass $m=4976 \mathrm{~kg}$ ), which is on a return leg from the Moon. It enters the atmosphere at $220 \mathrm{~km}$ altitude with a relative velocity of $V=$ $11 \mathrm{~km} / \mathrm{s}$, and a corresponding flight-path angle of $\gamma=-9.536^{\circ}$, see Fig. 2, top, for the reference trajectory. To avoid excessive fuel consumption for attitude control, the $z$-location of the c.o.m. has been moved down from the symmetry axis to provide a natural hypersonic trim condition with an angle of attack of about $\alpha=-24.5^{\circ}$. Angle-of-attack control is therefore only used to stabilize the angle of attack around this trim condition; bank-angle-control is used to orient the lift in a lift-down conditon to avoid skipping flight, and is effectively the only guidance means. Since at this point no guidance system is yet available we will take the attitude profiles from an open-loop, 6 degrees-of-freedom simulation (Fig. 2, bottom), smooth them and assume them to be the (nominal) guidance commands, where the angle of sideslip has been put to zero.
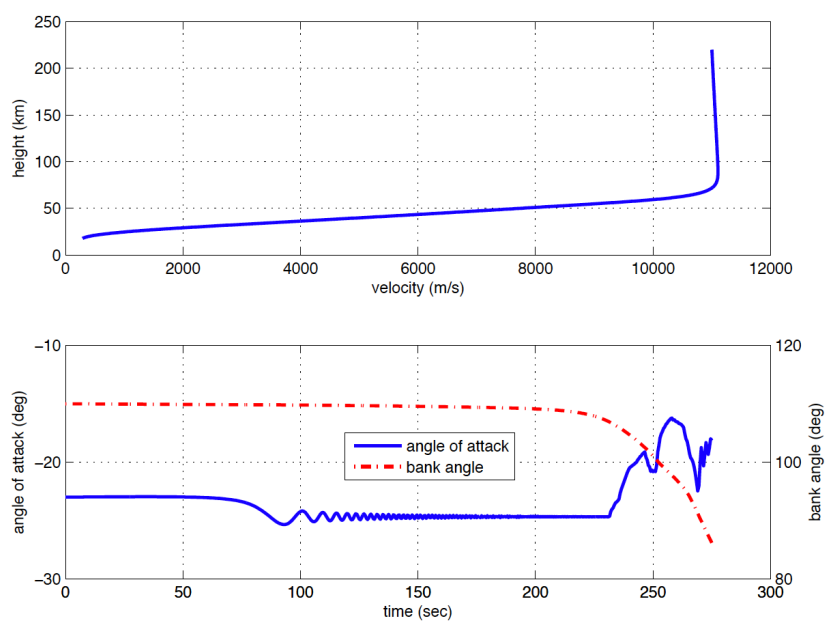

Figure 2. Reference trajectory (top) and attitude profile (bottom) for a free-fall entry.

The motion of re-entry vehicles is mainly driven by the combination of aerodynamic and gravitational forces and moments. Depending on the inherent non-linearities in the vehicles aerodynamic characteristics, the extent of the flight regime under consideration, and asymmetry in the mass distribution of the vehicle, this motion can only be accurately described by a set of coupled first-order non-linear differential equations. To describe the translational motion of a rigid body, a set of 6 differential equations is required. The form of these equations depends on the selected state variables, but in general they can be found in many textbooks. In this paper we use spherical components for position (distance $R$, longitude $\tau$, and latitude $\delta$ ) and velocity (velocity $V$, flight-path angle $\gamma$ and heading $\chi$ ). ${ }^{13,14}$ The state vector is thus given by $\mathbf{x}=(V, \gamma, \chi, R, \tau, \delta)^{T}$ and $\mathbf{u}=(\alpha, \sigma)^{T}$. In case of guidance, the control vector $\mathbf{u}$ includes the commanded angle of attack, $\alpha$, and bank angle, $\sigma$, which will be determined by the guidance system. The corresponding equations of motion read:

$$
\begin{gathered}
\dot{V}=-\frac{D}{m}+g \sin \gamma+\omega_{c b}^{2} R \cos \delta(\sin \gamma \cos \delta-\cos \gamma \sin \delta \cos \chi) \\
V \dot{\gamma}=\frac{L \cos \sigma}{m}-g \cos \gamma+2 \omega_{c b} V \cos \delta \sin \chi+\frac{V^{2}}{R} \cos \gamma+\omega_{c b}^{2} R \cos \delta(\cos \delta \cos \gamma+\sin \gamma \sin \delta \cos \chi) \\
V \cos \gamma \dot{\chi}=\frac{L \sin \sigma}{m}+2 \omega_{c b} V(\sin \delta \cos \gamma-\cos \delta \sin \gamma \cos \chi)+\frac{V^{2}}{R} \cos ^{2} \gamma \tan \delta \sin \chi+\omega_{c b}^{2} R \cos \delta \sin \delta \sin \chi \\
\dot{R}=\dot{h}=V \sin \gamma \\
\dot{\tau}=\frac{V \sin \chi \cos \gamma}{R \cos \delta} \\
\dot{\delta}=\frac{V \cos \chi \cos \gamma}{R}
\end{gathered}
$$


In the above equations, $\omega_{c b}$ is the rotational rate of the Earth, and $D$ and $L$ are the drag and lift force, respectively. Note that $\gamma$ is negative when the velocity is below the local horizon, and $\chi=0^{\circ}$ when flying to the north.

The rotational motion of entry capsules is mainly driven by the combination of aerodynamic and thruster moments. To describe the rotational motion of a rigid body, a set of 6 differential equations is required as well. In this paper we use a set of Euler angles for the attitude definition, namely the angle of attack, angle of sideslip and bank angle, in this case related to the groundspeed rather than the airspeed of the capsule. Since we will need the equations of rotational motion for the subsequent passivity analysis, they will be discussed more elaborately. The starting point for our model setup is a set of equations in the form:

$$
\dot{\mathbf{x}}=\mathbf{f}(\mathbf{x})+\mathbf{g}(\mathbf{x}, \mathbf{u}) \quad \mathbf{y}=\mathbf{h}(\mathbf{x})=\mathbf{C x}
$$

where $\mathbf{f}, \mathbf{g}$ and $\mathbf{h}$ can be determined from the general formulation of the equations of motion. ${ }^{8}$

Let us begin with the most general case of full state tracking. In Eq. $(29), \mathbf{x}=(p, q, r, \alpha, \beta, \sigma)^{T}$ and $\mathbf{u}=\left(M_{T, x}, M_{T, y}, M_{T, z}\right)^{T}$. The control vector $\mathbf{u}$ includes the three RCS thruster moments around the $X, Y$ and $Z$-axis of the body frame, respectively. These thruster-moments are independent of the vehicle state, so $\mathbf{g}(\mathbf{x}, \mathbf{u})=\mathbf{g}(\mathbf{u})$. To determine the functionals $\mathbf{f}$ and $\mathbf{g}$, we begin with the Euler equations of rotational motion:

$$
\dot{\omega}=\mathbf{I}^{-1}\left(\tilde{\mathbf{M}}_{\mathrm{cm}}-\boldsymbol{\omega} \times \mathbf{I} \boldsymbol{\omega}\right)
$$

with

$$
\begin{aligned}
& \tilde{\mathbf{M}}_{\mathbf{c m}}=\left(M_{x}, M_{y}, M_{z}\right)^{T}=\begin{array}{rr}
= & \text { sum of external moments about the c.o.m., expressed in compo- } \\
\text { nents along the body axes }
\end{array} \\
& \mathbf{I}=\left[\begin{array}{rrr}
I_{x x} & -I_{x y} & -I_{x z} \\
-I_{x y} & I_{y y} & -I_{y z} \\
-I_{x z} & -I_{y z} & I_{z z}
\end{array}\right]=\begin{array}{l}
\text { inertia tensor of the re-entry vehicle, referenced to the body frame } \\
\boldsymbol{\omega}=(p, q, r)^{T}
\end{array} \\
& =\begin{array}{l}
\text { the rotation vector of the body frame with respect to the inertial } \\
\text { frame, expressed in components along the body axes (roll rate } p, \\
\text { pitch rate } q \text { and yaw rate } r \text { ) }
\end{array}
\end{aligned}
$$

Solving the above equations requires the inversion of the inertia tensor. However, in a number of cases, these equations can be simplified. In the case of a mass-symmetrical vehicle, two out of three products of inertia are equal to zero (or all three, when the vehicle has two symmetry planes). When the plane of symmetry is the $X_{B} Z_{B}$-plane, which is the case since the location of the c.o.m. is only shifted down in $Z_{B}$-direction, then $I_{x y}=I_{y z}=0$. Eq. (30) can in that case be written as the following three scalar equations:

$$
\begin{gathered}
\dot{p}=\frac{I_{z z}}{I^{*}} M_{x}+\frac{I_{x z}}{I^{*}} M_{z}+\frac{\left(I_{x x}-I_{y y}+I_{z z}\right) I_{x z}}{I^{*}} p q+\frac{\left(I_{y y}-I_{z z}\right) I_{z z}-I_{x z}^{2}}{I^{*}} q r \\
\dot{q}=\frac{M_{y}}{I_{y y}}+\frac{I_{x z}}{I_{y y}}\left(r^{2}-p^{2}\right)+\frac{I_{z z}-I_{x x}}{I_{y y}} p r \\
\dot{r}=\frac{I_{x z}}{I^{*}} M_{x}+\frac{I_{x x}}{I^{*}} M_{z}+\frac{\left(I_{x x}-I_{y y}\right) I_{x x}+I_{x z}^{2}}{I^{*}} p q+\frac{\left(-I_{x x}+I_{y y}-I_{z z}\right) I_{x z}}{I^{*}} q r
\end{gathered}
$$

with $I^{*}=I_{x x} I_{z z}-I_{x z}^{2}$. The external moments $M_{x}, M_{y}$ and $M_{z}$ are the sum of the aerodynamic moments and the input from the RCS thrusters. Moreover, since in the original aerodynamic database ${ }^{15}$ the moment reference point is not the c.o.m, but the apex of the capsule, the aerodynamic moments also include a component due to the aerodynamic forces. Since we need explicit expressions for these moments, we will first compute the total aerodynamic moment and then use regression analysis to find these expressions.

The kinematic attitude equations are given by: ${ }^{14}$ 


$$
\begin{aligned}
\dot{\alpha} \cos \beta= & -p \cos \alpha \sin \beta+q \cos \beta-r \sin \alpha \sin \beta+ \\
& +\sin \sigma\left[\dot{\chi} \cos \gamma-\dot{\delta} \sin \chi \sin \gamma+\left(\dot{\tau}+\omega_{c b}\right)(\cos \delta \cos \chi \sin \gamma-\sin \delta \cos \gamma)\right]+ \\
& \quad-\cos \sigma\left[\dot{\gamma}-\dot{\delta} \cos \chi-\left(\dot{\tau}+\omega_{c b}\right) \cos \delta \sin \chi\right] \\
\dot{\beta}= & p \sin \alpha-r \cos \alpha+\sin \sigma\left[\dot{\gamma}-\dot{\delta} \cos \chi-\left(\dot{\tau}+\omega_{c b}\right) \cos \delta \sin \chi\right]+ \\
+ & \cos \sigma\left[\dot{\chi} \cos \gamma-\dot{\delta} \sin \chi \sin \gamma+\left(\dot{\tau}+\omega_{c b}\right)(\cos \delta \cos \chi \sin \gamma-\sin \delta \cos \gamma)\right] \\
\dot{\sigma}= & -p \cos \alpha \cos \beta-q \sin \beta-r \sin \alpha \cos \beta+ \\
& +\dot{\alpha} \sin \beta-\dot{\chi} \sin \gamma-\dot{\delta} \sin \chi \cos \gamma+\left(\dot{\tau}+\omega_{c b}\right)(\cos \delta \cos \chi \cos \gamma+\sin \delta \sin \gamma)
\end{aligned}
$$

In these equations, $\dot{\gamma}, \dot{\chi}, \dot{\tau}$ and $\dot{\delta}$ are related to the rotation of the local horizontal plane, as a consequence of the sphericity of the Earth. However, when considering attitude motion that is expected to be at least one order of magnitude faster than the translational motion, it is allowed to consider a "frozen" local horizontal plane. This effectively puts $\dot{\gamma}, \dot{\chi}, \dot{\tau}$ and $\dot{\delta}$ to zero.

Rewriting the dynamic and kinematic equations of motion in the form of Eq. (29) yields for the functionals f and $g$ :

$$
\begin{gathered}
f_{1}(\mathbf{x})=\frac{\left(I_{x x}-I_{y y}+I_{z z}\right) I_{x z}}{I^{*}} p q+\frac{\left(I_{y y}-I_{z z}\right) I_{z z}-I_{x z}^{2}}{I^{*}} q r+\frac{I_{z z}}{I^{*}} \mathcal{L}(h, V, \beta)+\frac{I_{x z}}{I^{*}} \mathcal{N}(h, V, r, \beta) \\
f_{2}(\mathbf{x})=\frac{I_{x z}}{I_{y y}}\left(r^{2}-p^{2}\right)+\frac{I_{z z}-I_{x x}}{I_{y y}} p r+\frac{\mathcal{M}(h, V, q, \alpha)}{I_{y y}} \\
f_{3}(\mathbf{x})=\frac{\left(I_{x x}-I_{y y}\right) I_{x x}+I_{x z}^{2}}{I^{*}} p q+\frac{\left(-I_{x x}+I_{y y}-I_{z z}\right) I_{x z}}{I^{*}} q r+\frac{I_{x z}}{I^{*}} \mathcal{L}(h, V, \beta)+\frac{I_{x x}}{I^{*}} \mathcal{N}(h, V, r, \beta) \\
f_{4}(\mathbf{x})=-p \cos \alpha \tan \beta+q-r \sin \alpha \tan \beta \\
f_{5}(\mathbf{x})=p \sin \alpha-r \cos \alpha \\
f_{6}(\mathbf{x})=-p \cos \alpha \cos \beta-q \sin \beta-r \sin \alpha \cos \beta+f_{4} \sin \beta \\
g_{1}(\mathbf{x}, \mathbf{u})=\frac{I_{z z}}{I^{*}} M_{T, x}+\frac{I_{x z}}{I^{*}} M_{T, z} \\
g_{2}(\mathbf{x}, \mathbf{u})=\frac{M_{T, y}}{I_{y y}} \\
g_{3}(\mathbf{x}, \mathbf{u})=\frac{I_{x z}}{I^{*}} M_{T, x}+\frac{I_{x x}}{I^{*}} M_{T, z} \\
g_{4}=g_{5}=g_{6}=0
\end{gathered}
$$

In the functionals $f_{1}, f_{2}$ and $f_{3}$, the aerodynamic roll, pitch and yaw moments, $\mathcal{L}, \mathcal{M}$ and $\mathcal{N}$, appear that are functions of the states $h, V, \alpha, \beta, q$ and $r:{ }^{15,16}$ 


$$
\begin{gathered}
\mathcal{L}(h, V, \alpha, \beta)=C_{l}(M(h, V), \alpha, \beta) \frac{1}{2} \rho(h) V^{2} S d \\
\mathcal{M}(h, V, \alpha, \beta)=\left(C_{m}(M(h, V), \alpha, \beta)+C_{m_{q}}(M(h, V), \alpha, \beta) \frac{q d}{V}\right) \frac{1}{2} \rho(h) V^{2} S d \\
\mathcal{N}(h, V, \alpha, \beta)=\left(C_{n}(M(h, V), \alpha, \beta)+C_{n_{r}}(M(h, V), \alpha, \beta) \frac{r d}{V}\right) \frac{1}{2} \rho(h) V^{2} S d
\end{gathered}
$$

where $\rho(h)$ is the altitude-dependent atmospheric density and $M(h, V)$ is the Mach number, through the speed-of-sound dependency with altitude (through the temperature) a function of both $h$ and $V . S$ and $d$ are the aerodynamic reference area and length (i.e., the vehicle diameter), respectively. To derive expressions for the aerodynamic coefficients over the complete flight range will lead to very complex expressions. In our approach we have assumed that the rotational motion is decoupled from the translational motion, which removes any velocity and altitude dependency. This yields design points with a constant Mach number, atmospheric density and dynamic pressure. The moment equations (47)-(49) can thus be simplified to:

$$
\begin{gathered}
\mathcal{L}(\alpha, \beta)=C_{l}(\alpha, \beta) \bar{q}_{e} S d \\
\mathcal{M}(\alpha, \beta, q)=\left(C_{m}(\alpha, \beta)+C_{m_{q}}(\alpha, \beta) \frac{q d}{V_{e}}\right) \bar{q}_{e} S d \\
\mathcal{N}(\alpha, \beta, r)=\left(C_{n}(\alpha, \beta)+C_{n_{r}}(\alpha, \beta) \frac{r d}{V_{e}}\right) \bar{q}_{e} S d
\end{gathered}
$$

In the above equations, the subscript $e$ indicates the design point's equilibrium state for translational motion; $\bar{q}_{e}=\frac{1}{2} \rho\left(h_{e}\right) V_{e}^{2}$ is the dynamic pressure.

For each of the design points the functionals $f_{1}, f_{2}$ and $f_{3}$ in Eqs. (37) through (39) are therefore expressed by

$$
\begin{gathered}
f_{1}(\mathbf{x})=\frac{\left(I_{x x}-I_{y y}+I_{z z}\right) I_{x z}}{I^{*}} p q+\frac{\left(I_{y y}-I_{z z}\right) I_{z z}-I_{x z}^{2}}{I^{*}} q r+\frac{I_{z z}}{I^{*}} \mathcal{L}(\alpha, \beta)+\frac{I_{x z}}{I^{*}} \mathcal{N}(\alpha, \beta, r) \\
f_{2}(\mathbf{x})=\frac{I_{x z}}{I_{y y}}\left(r^{2}-p^{2}\right)+\frac{I_{z z}-I_{x x}}{I_{y y}} p r+\frac{\mathcal{M}(\alpha, \beta, q)}{I_{y y}} \\
f_{3}(\mathbf{x})=\frac{\left(I_{x x}-I_{y y}\right) I_{x x}+I_{x z}^{2}}{I^{*}} p q+\frac{\left(-I_{x x}+I_{y y}-I_{z z}\right) I_{x z}}{I^{*}} q r+\frac{I_{x z}}{I^{*}} \mathcal{L}(\alpha, \beta)+\frac{I_{x x}}{I^{*}} \mathcal{N}(\alpha, \beta, r)
\end{gathered}
$$

Furthermore, after inspecting the aerodynamic database more carefully shows that there is not so much variation in the coefficients for Mach numbers larger than $M=3.4$ for the angle-of-attack and angle-ofsideslip range of interest. Therefore, we can assume Mach-independent aerodynamic properties for $M \geq 3.4$ and $\alpha \in\left[-30^{\circ},-15^{\circ}\right]$ and $\beta \in\left[-5^{\circ}, 5^{\circ}\right]$, which means that we can use the same aerodynamic model for all Mach numbers from entry down to $M=3.4$. For each lower Mach number - or an extension of the attitude-angle range - a separate model should be derived; this remains to be done as future work. Note that as mentioned earlier, the contribution of the aerodynamic forces to the moment will be included in the regression model for the moment coefficients.

The following regression models are derived (with $\alpha$ and $\beta$ in radians):

$$
\begin{gathered}
C_{l}=0.01 \beta+0.01 \alpha \beta-0.08 \alpha^{2} \beta \\
C_{m}=0.12+1.25 \alpha+3.64 \alpha^{2}+3.18 \alpha^{3}+1.55 \alpha \beta^{2}+0.91 \alpha^{2} \beta^{2} \\
C_{n}=1.75 \beta-0.16 \alpha \beta-1.80 \alpha^{2} \beta \\
C_{l_{p}}=0 \\
C_{m_{q}}=-0.010-1.021 \alpha \beta^{2}-3.960 \alpha^{2} \beta^{2}-4.031 \alpha^{3} \beta^{2} \\
C_{n_{r}}=0.06 \beta--0.35 \alpha^{2} \beta-4.06 \alpha^{2} \beta^{3}-0.43 \alpha^{3} \beta-8.21 \alpha^{3} \beta^{3}
\end{gathered}
$$

Substituting the above expressions in Eqs. (53)-(55) yields: 


$$
\begin{aligned}
f_{1}(\mathbf{x})= & \frac{\left(I_{x x}-I_{y y}+I_{z z}\right) I_{x z}}{I^{*}} p q+\frac{\left(I_{y y}-I_{z z}\right) I_{z z}-I_{x z}^{2}}{I^{*}} q r+ \\
+ & \frac{I_{z z}}{I^{*}}\left(0.01+0.01 \alpha-0.08 \alpha^{2}\right) \beta \bar{q}_{e} S d+\frac{I_{x z}}{I^{*}}\left(1.75-0.16 \alpha-1.80 \alpha^{2}\right) \beta \bar{q}_{e} S d+ \\
+ & \frac{I_{x z}}{I^{*}}\left(0.06-0.35 \alpha^{2}-4.06 \alpha^{2} \beta^{2}-0.43 \alpha^{3}-8.21 \alpha^{3} \beta^{2}\right) \beta \frac{r d}{V_{e}} \bar{q}_{e} S d \\
f_{2}(\mathbf{x})= & \frac{I_{x z}}{I_{y y}}\left(r^{2}-p^{2}\right)+\frac{I_{z z}-I_{x x}}{I_{y y}} p r+ \\
& +\frac{1}{I_{y y}}\left(0.12+1.25 \alpha+3.64 \alpha^{2}+3.18 \alpha^{3}+1.55 \alpha \beta^{2}+0.91 \alpha^{2} \beta^{2}\right) \bar{q}_{e} S d+ \\
& +\frac{1}{I_{y y}}\left(-0.010-1.021 \alpha \beta^{2}-3.960 \alpha^{2} \beta^{2}-4.031 \alpha^{3} \beta^{2}\right) \frac{q d}{V_{e}} \bar{q}_{e} S d \\
f_{3}(\mathbf{x})= & \frac{\left(I_{x x}-I_{y y}\right) I_{x x}+I_{x z}^{2}}{I^{*}} p q+\frac{\left(-I_{x x}+I_{y y}-I_{z z}\right) I_{x z}}{I^{*}} q r+ \\
+ & \frac{I_{x z}}{I^{*}}\left(0.01+0.01 \alpha-0.08 \alpha^{2}\right) \beta \bar{q}_{e} S d+\frac{I_{x x}}{I^{*}}\left(1.75-0.16 \alpha-1.80 \alpha^{2}\right) \beta \bar{q}_{e} S d+ \\
+ & \frac{I_{x x}}{I^{*}}\left(0.06-0.35 \alpha^{2}-4.06 \alpha^{2} \beta^{2}-0.43 \alpha^{3}-8.21 \alpha^{3} \beta^{2}\right) \beta \frac{r d}{V_{e}} \bar{q}_{e} S d
\end{aligned}
$$

Finally, Eqs. (62)-(64), together with Eqs. (40)-(46) mapped on Eq. (13) gives us:

$$
\left(\begin{array}{c}
\dot{p} \\
\dot{q} \\
\dot{r} \\
\dot{\alpha} \\
\dot{\beta} \\
\dot{\sigma}
\end{array}\right)=\mathbf{A}_{\mathbf{p}}\left(\begin{array}{c}
p \\
q \\
r \\
\alpha \\
\beta \\
\sigma
\end{array}\right)+\mathbf{B}_{\mathbf{p}}\left(\begin{array}{c}
M_{T, x} \\
M_{T, y} \\
M_{T, z}
\end{array}\right)
$$

Here,

with

$$
\mathbf{A}_{\mathbf{p}}=\left[\begin{array}{cccccc}
a_{p p} & 0 & a_{p r} & 0 & a_{p \beta} & 0 \\
a_{q p} & a_{q q} & a_{q r} & a_{q \alpha} & 0 & 0 \\
a_{r p} & 0 & a_{r r} & 0 & a_{r \beta} & 0 \\
a_{\alpha p} & a_{\alpha q} & a_{\alpha r} & 0 & 0 & 0 \\
a_{\beta p} & 0 & a_{\beta r} & 0 & 0 & 0 \\
a_{\sigma p} & 0 & a_{\sigma r} & 0 & 0 & 0
\end{array}\right]
$$

$$
\begin{gathered}
a_{p p}=\frac{\left(I_{x x}-I_{y y}+I_{z z}\right) I_{x z}}{I^{*}} q \\
a_{p r}=\frac{\left(I_{y y}-I_{z z}\right) I_{z z}-I_{x z}^{2}}{I^{*}} q \\
a_{p \beta}=\frac{I_{z z}}{I^{*}}\left(0.01+0.01 \alpha-0.08 \alpha^{2}\right) \bar{q}_{e} S d+\frac{I_{x z}}{I^{*}}\left(1.75-0.16 \alpha-1.80 \alpha^{2}\right) \bar{q}_{e} S d+ \\
+\frac{I_{x z}}{I^{*}}\left(0.06-0.35 \alpha^{2}-4.06 \alpha^{2} \beta^{2}-0.43 \alpha^{3}-8.21 \alpha^{3} \beta^{2}\right) \frac{r d}{V_{e}} \bar{q}_{e} S d \\
a_{q p}=-\frac{I_{x z}}{I_{y y}} p \\
a_{q q}=\frac{1}{I_{y y}}\left(-0.010-1.021 \alpha \beta^{2}-3.960 \alpha^{2} \beta^{2}-4.031 \alpha^{3} \beta^{2}\right) \frac{\bar{q}_{e} S d^{2}}{V_{e}}
\end{gathered}
$$




$$
\begin{gathered}
a_{q r}=\frac{I_{x z}}{I_{y y}} r+\frac{I_{z z}-I_{x x}}{I_{y y}} p \\
a_{q \alpha}=\frac{1}{I_{y y}}\left(\frac{0.12}{\alpha}+1.25+3.64 \alpha+3.18 \alpha^{2}+1.55 \beta^{2}+0.91 \alpha \beta^{2}\right) \bar{q}_{e} S d \\
a_{r p}=\frac{\left(I_{x x}-I_{y y}\right) I_{x x}+I_{x z}^{2}}{I^{*}} q \\
a_{r r}=\frac{\left(-I_{x x}+I_{y y}-I_{z z}\right) I_{x z}}{I^{*}} q \\
a_{r \beta}=\frac{I_{x z}}{I^{*}}\left(0.01+0.01 \alpha-0.08 \alpha^{2}\right) \bar{q}_{e} S d+\frac{I_{x x}}{I^{*}}\left(1.75-0.16 \alpha-1.80 \alpha^{2}\right) \bar{q}_{e} S d+ \\
+\frac{I_{x x}}{I^{*}}\left(0.06-0.35 \alpha^{2}-4.06 \alpha^{2} \beta^{2}-0.43 \alpha^{3}-8.21 \alpha^{3} \beta^{2}\right) \frac{r d}{V_{e}} \bar{q}_{e} S d \\
a_{\alpha p}=-\cos \alpha \tan \beta \\
a_{\alpha q}=1 \\
a_{\alpha r}=-\sin \alpha \tan \beta \\
a_{\beta p}=\sin \alpha \\
a_{\beta r}=-\cos \alpha \\
a_{\sigma p}=-\frac{\cos \alpha}{\cos \beta} \\
a_{\sigma r}=-\frac{\sin \alpha}{\cos \beta}
\end{gathered}
$$

and

$$
\mathbf{B}_{\mathbf{p}}=\left[\begin{array}{ccc}
\frac{I_{z z}}{I^{*}} & 0 & \frac{I_{x z}}{I^{*}} \\
0 & \frac{1}{I_{y y}} & 0 \\
\frac{I_{x z}}{I^{*}} & 0 & \frac{I_{x x}}{I^{*}} \\
0 & 0 & 0 \\
0 & 0 & 0 \\
0 & 0 & 0
\end{array}\right]
$$

Note that the definition of $\mathbf{A}_{\mathbf{p}}$ is only one out of more possible definitions, depending on how the coefficients are extracted from the functionals $\mathbf{f}$ and $\mathbf{g}$.

Next, we need to set up the output equation of Eq. (29). Full state feedback requires that $\mathbf{C}_{\mathbf{p}}\left(\mathbf{x}_{\mathbf{p}}, t\right)$ is the $6 \mathrm{x} 6$ identity matrix. However, as the adaptive controller requires only three outputs to compute the three thruster moments, per input-output channel the six states will be combined to improve the damping and the performance of the closed-loop system. This is best done according to the optimal gain matrix $\mathbf{K}$ that follows from solving the Algebraic Riccati Equation, ${ }^{8,12}$ yielding $\mathbf{y}_{\mathbf{p}}(t)=\mathbf{C}_{\mathbf{p}}\left(\mathbf{x}_{\mathbf{p}}, t\right) \mathbf{x}_{\mathbf{p}}(t)=\mathbf{K}\left(\mathbf{x}_{\mathbf{p}}, t\right) \mathbf{x}_{\mathbf{p}}(t)$. In case we neglect the smaller terms in $\mathbf{K}\left(\mathbf{x}_{\mathbf{p}}, t\right)$ and put them to zero, the longitudinal and lateral motion will be decoupled, which is allowed for the control-system design. That means that for complete state measurement $\mathbf{C}_{\mathbf{p}}\left(\mathbf{x}_{\mathbf{p}}, t\right)$ changes to:

$$
\mathbf{C}_{\mathbf{p}}\left(\mathbf{x}_{\mathbf{p}}, t\right)=\left[\begin{array}{cccccc}
K_{p p} & 0 & K_{p r} & 0 & K_{p \beta} & K_{p \sigma} \\
0 & K_{q q} & 0 & K_{q \alpha} & 0 & 0 \\
K_{r p} & 0 & K_{r r} & 0 & K_{r \beta} & K_{r \sigma}
\end{array}\right]
$$

In Fig. 3 the time history of the optimal gains is plotted. Note the change in angle-of-attack and sidesliprelated gains around $t \approx 100 \mathrm{~s}$. At this moment the dynamic pressure is sufficiently large to induce angleof-attack and angle-of-sideslip oscillations (see Fig. 2). With ever increasing dynamic pressure the thrusters need to counteract growing aerodynamic moments, which requires larger thruster gains. 

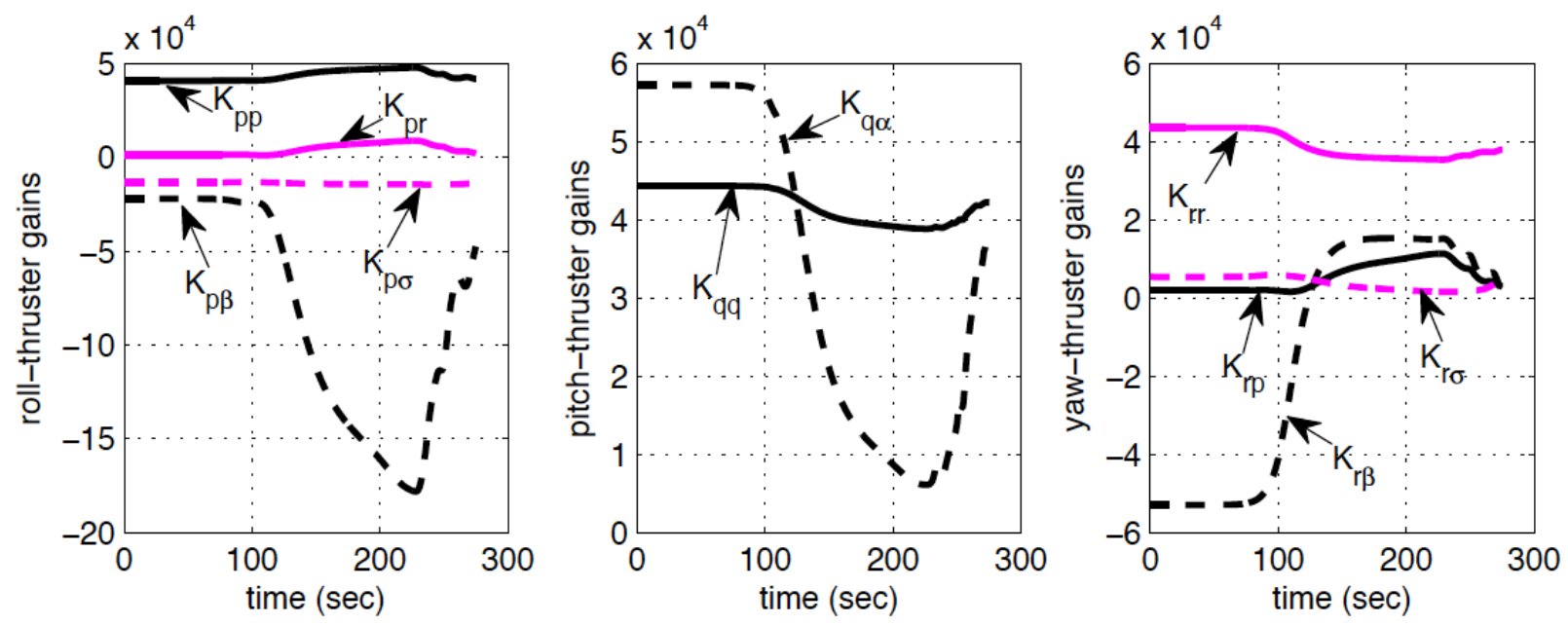

Figure 3. Time history of the optimal gains for the roll (left), pitch (middle) and yaw (right) thrusters.

\section{Passivity Analysis}

\section{A. Linear System}

As a first step in the analysis we consider an LTI version of the model given by Eqs. (37)-(46), to see whether the LTI system fulfills the ASPR requirements Eq. (11)-(12). Linearizing Eqs. (37)-(46) and realizing that $I_{x z}$ cannot be neglected with respect to $I_{x x}, I_{y y}$ and $I_{z z}$ we obtain the following state-space system of equations, where we have retained only the first derivatives of the aerodynamic moments:

$$
\Delta \dot{\mathbf{x}}=\mathbf{A}_{\mathbf{m}} \Delta \mathbf{x}+\mathbf{B}_{\mathbf{m}} \Delta \mathbf{u}
$$

with

$$
\begin{aligned}
& \mathbf{A}_{\mathbf{m}}=\left[\begin{array}{cccc}
\frac{\left(I_{x x}-I_{y y}+I_{z z}\right) I_{x z}}{I^{*}} q_{e} & \frac{\left(I_{x x}-I_{y y}+I_{z z}\right) I_{x z}}{I^{*}} p_{e}+\frac{\left(I_{y y}-I_{z z}\right) I_{z z}-I_{x z}^{2}}{I^{*}} r_{e} & \frac{\left(I_{y y}-I_{z z}\right) I_{z z}-I_{x z}^{2}}{I_{z}} q_{e} & \ldots \\
-2 \frac{I_{x x}}{I_{y y}} p_{e}+\frac{I_{z z}}{I_{y y}} r_{e} & 0 & \frac{I_{z z}-I_{x x}}{I_{y y}} p_{e}+2 \frac{I_{x z}}{I_{y y}} r_{e} & \ldots \\
\frac{\left(I_{x x}-I_{y y}\right) I_{x x}+I_{x z}}{I^{*}} q_{e} & \frac{\left(I_{x x}-I_{y y}\right) I_{x x}+I_{x z}^{2}}{I^{*}} p_{e}+\frac{\left(-I_{x x}+I_{y y}-I_{z z}\right) I_{x z}}{I^{*}} r_{e} & \frac{\left(-I_{x x}+I_{y y}-I_{z z}\right) I_{x z}}{I^{*}} q_{e} & \ldots \\
0 & 1 & 0 & \ldots \\
\sin \alpha_{e} & 0 & -\cos \alpha_{e} & \ldots \\
-\cos \alpha_{e} & 0 & -\sin \alpha_{e} & \ldots
\end{array}\right. \\
& \left.\begin{array}{lccc}
\ldots & 0 & \left(\frac{I_{z z}}{I^{*}} \frac{\partial C_{l}}{\partial \beta}+\frac{I_{x z}}{I^{*}} \frac{\partial C_{n}}{\partial \beta}\right) \bar{q}_{e} S d & 0 \\
\ldots & \frac{1}{I_{y y}} \frac{\partial C_{m}}{\partial \alpha} \bar{q}_{e} S d & 0 & 0 \\
\ldots & 0 & \left(\frac{I_{x z}}{I^{*}} \frac{\partial C_{l}}{\partial \beta}+\frac{I_{x x}}{I^{*}} \frac{\partial C_{n}}{\partial \beta}\right) \bar{q}_{e} S d & 0 \\
\ldots & 0 & 0 & 0 \\
\ldots & 0 & 0 & 0 \\
\ldots & 0 & 0 & 0
\end{array}\right]
\end{aligned}
$$

and $\mathbf{B}_{\mathbf{m}}=\mathbf{B}_{\mathbf{p}}$, see Eq. (67). The nominal angular velocities $p_{e}, q_{e}$ and $r_{e}$ are derived from the requirement $\dot{\alpha}=\dot{\beta}=\dot{\sigma}=0^{\circ}$, i.e.,

$$
\begin{gathered}
p_{e}=c_{1} \sin \alpha_{e}+c_{2} \cos \alpha_{e} \\
q_{e}=\frac{L_{e}}{m V_{e}}-\frac{g_{e}}{V_{e}} \cos \gamma_{e} \cos \sigma_{e}
\end{gathered}
$$



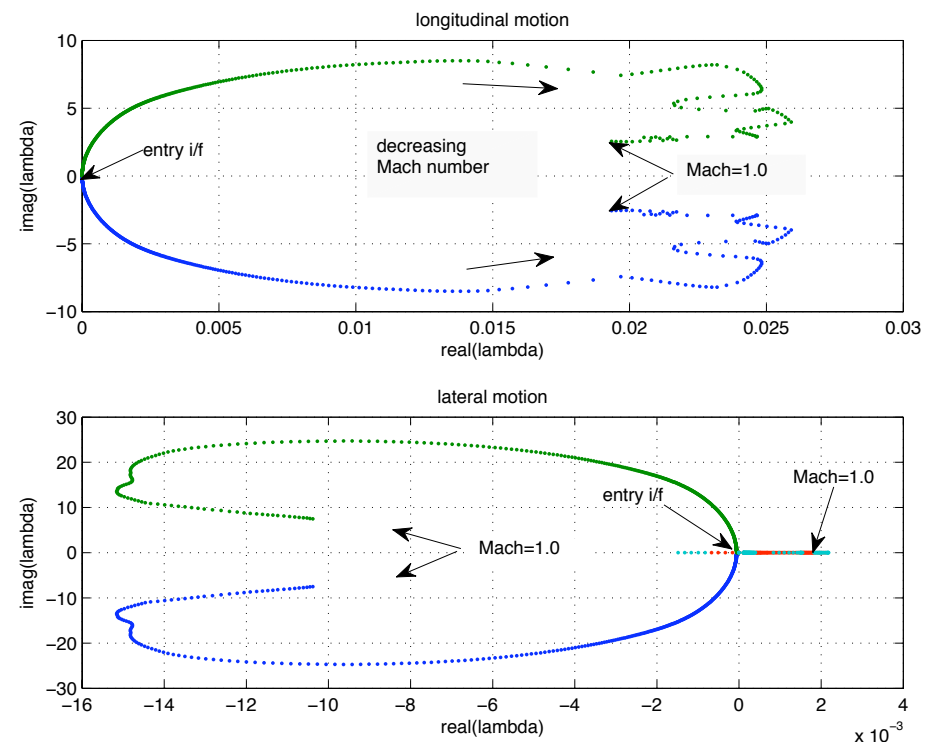

Figure 4. Eigenvalues for the longitudinal motion (top) and lateral motion (bottom). Evaluation done for discrete time points.

$$
r_{e}=-c_{1} \cos \alpha_{e}+c_{2} \sin \alpha_{e}
$$

with $c_{1}=\frac{g_{e}}{V_{e}} \cos \gamma_{e} \sin \sigma_{e}$ and $c_{2}=\frac{L_{e}}{m V_{e}} \tan \gamma_{e} \sin \sigma_{e}{ }^{14}$

To have some more insight in the system at hand, in Fig. 4 the variation of eigenvalues of the entry capsule has been plotted as a function of flight time. It is clear that the open-loop longitudinal motion of the linearized system is marginally unstable right from the moment the capsule is entering the atmosphere. With decreasing Mach number the eigenvalues become more positive, although not so much. The lateral motion consists of a lightly damped oscillatory motion and two aperiodic motions that are marginally unstable. Summarized, this type of open-loop behavior should not pose any problems for any attitude controller.

Checking for $\mathbf{C}_{\mathbf{p}} \mathbf{B}_{\mathbf{p}}>0$ confirms that for the output matrix Eq. (68) this condition is fulfilled throughout the flight (i.e., the non-symmetric matrix product is diagonizable). Also the zero dynamics is stable, see Fig. 5. It is shown that the real part of the zero dynamics is negative, although it becomes less negative towards the end of the flight. The erratic behavior is due to the relatively abrupt angle-of-attack excursions, see also Fig. 2. So, in conclusion, since the LTI fulfills both sufficient conditions it is ASPR with the current definition of the output matrix.

For the current analysis we have recalculated the optimal gain matrix $\mathbf{K}\left(\mathbf{x}_{\mathbf{p}}, t\right)$ for each time point. However, a simple analysis shows that if we keep the gain matrix (and thus $\mathbf{C}_{\mathbf{p}}\left(\mathbf{x}_{\mathbf{p}}, t\right)$ ) constant, the LTI system is still ASPR. This means that the selection of the proper output matrix is not so critical, although the right combination of angular rate and attitude angles should be taken. On the other hand, if we would only feed back the angular rates the zero dynamics is only partially stable, which means that the LTI system is not always ASPR. Finally, if only the attitude angles are fed back the system is not ASPR.

\section{B. Non-Linear System}

For the analysis of the non-linear system, we will use again the output matrix Eq. (68), which led to $\mathbf{C}_{\mathbf{p}} \mathbf{B}_{\mathbf{p}}>0$ for the LTI system. To check the stability of the zero dynamics, Eq. (21), we need to define the matrices $\mathbf{N}_{\mathbf{n}-\mathbf{m}, \mathbf{n}}$ and $\mathbf{M}_{\mathbf{n}, \mathbf{n}-\mathbf{m}}$, such that the equalities of Eq. (19) are fulfilled. With 6 states $(n=6)$ and 3 RCS control moments $(m=3)$, Eq. (19) is fully written out as: 

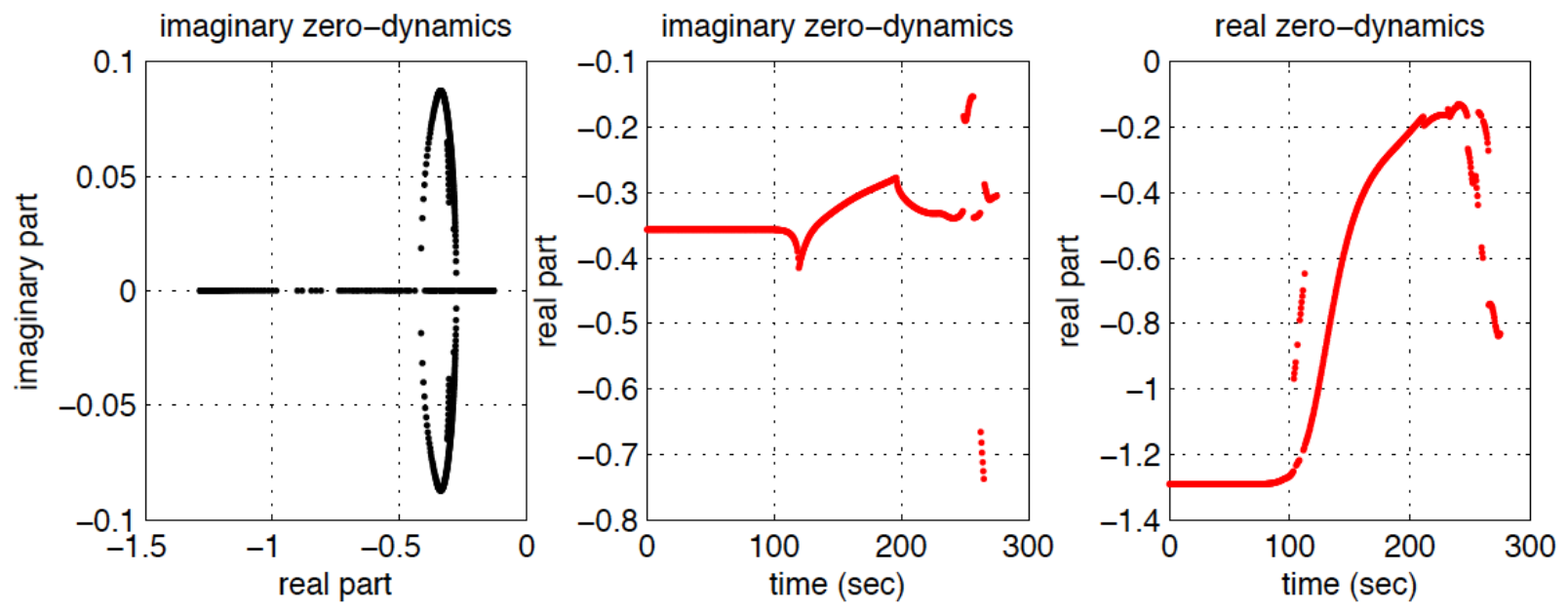

Figure 5. Zero dynamics for the LTI system $(n=6, m=3)$, evaluated at discrete time points: the imaginary pair (left) with the time history of the real part (middle), and the real component versus time (right).

$$
\begin{gathered}
{\left[\begin{array}{llllll}
n_{11} & n_{12} & n_{13} & n_{14} & n_{15} & n_{16} \\
n_{21} & n_{22} & n_{23} & n_{24} & n_{25} & n_{26} \\
n_{31} & n_{32} & n_{33} & n_{34} & n_{35} & n_{36}
\end{array}\right]\left[\begin{array}{lll}
m_{11} & m_{12} & m_{13} \\
m_{21} & m_{22} & m_{23} \\
m_{31} & m_{32} & m_{33} \\
m_{41} & m_{42} & m_{43} \\
m_{51} & m_{52} & m_{53} \\
m_{61} & m_{62} & m_{63}
\end{array}\right]=\left[\begin{array}{lll}
1 & 0 & 0 \\
0 & 1 & 0 \\
0 & 0 & 1
\end{array}\right]} \\
{\left[\begin{array}{cccccc}
c_{11} & 0 & c_{13} & 0 & c_{15} & c_{16} \\
0 & c_{22} & 0 & c_{14} & 0 & 0 \\
c_{31} & 0 & c_{33} & 0 & c_{34} & c_{36}
\end{array}\right]\left[\begin{array}{ccc}
m_{11} & m_{12} & m_{13} \\
m_{21} & m_{22} & m_{23} \\
m_{31} & m_{32} & m_{33} \\
m_{41} & m_{42} & m_{43} \\
m_{51} & m_{52} & m_{53} \\
m_{61} & m_{62} & m_{63}
\end{array}\right]=\left[\begin{array}{lll}
0 & 0 & 0 \\
0 & 0 & 0 \\
0 & 0 & 0
\end{array}\right]} \\
{\left[\begin{array}{cccccc}
n_{11} & n_{12} & n_{13} & n_{14} & n_{15} & n_{16} \\
n_{21} & n_{22} & n_{23} & n_{24} & n_{25} & n_{26} \\
n_{31} & n_{32} & n_{33} & n_{34} & n_{35} & n_{36}
\end{array}\right]\left[\begin{array}{ccc}
b_{11} & 0 & b_{13} \\
0 & b_{12} & 0 \\
b_{31} & 0 & b_{33} \\
0 & 0 & 0 \\
0 & 0 & 0 \\
0 & 0 & 0
\end{array}\right]=\left[\begin{array}{ccc}
0 & 0 & 0 \\
0 & 0 & 0 \\
0 & 0 & 0
\end{array}\right]}
\end{gathered}
$$

In the above equations we have adapted the notation $b_{i j}$ and $c_{i j}$ to indicate the non-zero elements of $\mathbf{B}_{\mathbf{p}}$ and $\mathbf{C}_{\mathbf{p}}$, respectively. With these 27 equations 36 coefficients have to be derived, which means that we have 9 extra degrees of freedom. So, we need to (cleverly) assume values for 9 coefficients before we can derive the remaining 27. This is not a trivial task, since not every combination that we tried resulted in a solution for the 27 coefficients. Below, we will summarize the approach that we followed to reach the final solution.

We begin with Eq. (76), since due to the many zero coefficients in $\mathbf{B}_{\mathbf{p}}$ it is relatively easy to isolate some $n_{i j}$. The individual scalar equations read:

$$
\begin{gathered}
n_{11} b_{11}+n_{13} b_{31}=0 \\
n_{12} b_{22}=0 \\
n_{11} b_{13}+n_{13} b_{33}=0
\end{gathered}
$$




$$
\begin{gathered}
n_{21} b_{11}+n_{23} b_{31}=0 \\
n_{22} b_{22}=0 \\
n_{21} b_{13}+n_{23} b_{33}=0 \\
n_{31} b_{11}+n_{33} b_{31}=0 \\
n_{32} b_{22}=0 \\
n_{31} b_{13}+n_{33} b_{33}=0
\end{gathered}
$$

From Eqs. (78), (81) and (84) it automatically follows that $n_{12}=n_{22}=n_{32}=0$. Eq. (77) yields $n_{11}=-\frac{b_{31}}{b_{11}} n_{13}$ and substituted in Eq. (79) gives

$$
\left(-\frac{b_{31} b_{13}}{b_{11}}+b_{33}\right) n_{13}=0
$$

This results in $n_{13}=0$, and thus also $n_{11}=0$. Similarly, we find also that $n_{21}=n_{23}=n_{31}=n_{33}=0$.

Eq. (75) written out gives:

$$
\begin{gathered}
c_{11} m_{11}+c_{13} m_{31}+c_{15} m_{51}+c_{16} m_{61}=0 \\
c_{11} m_{12}+c_{13} m_{32}+c_{15} m_{52}+c_{16} m_{62}=0 \\
c_{11} m_{13}+c_{13} m_{33}+c_{15} m_{53}+c_{16} m_{63}=0 \\
c_{22} m_{21}+c_{24} m_{41}=0 \\
c_{22} m_{22}+c_{24} m_{42}=0 \\
c_{22} m_{23}+c_{24} m_{43}=0 \\
c_{31} m_{11}+c_{33} m_{31}+c_{35} m_{51}+c_{36} m_{61}=0 \\
c_{31} m_{12}+c_{33} m_{32}+c_{35} m_{52}+c_{36} m_{62}=0 \\
c_{31} m_{13}+c_{33} m_{33}+c_{35} m_{53}+c_{36} m_{63}=0
\end{gathered}
$$

Assuming that $m_{21}=1, m_{22}=0$ and $m_{23}=1$, Eqs. (90)-(92) give us $m_{41}=-\frac{c_{22}}{c_{24}}, m_{42}=0$ and $m_{43}=-\frac{c_{22}}{c_{24}}$. From the remaining 6 equations we can solve $m_{32}, m_{33}, m_{51}, m_{53}, m_{61}$ and $m_{62}$, once we assume values for the remaining $6 m_{i j}$, i.e., $m_{11}=m_{12}=m_{13}=m_{31}=m_{52}=m_{63}=1$. The final result for $\mathbf{M}$ is now:

$$
\mathbf{M}=\left[\begin{array}{ccc}
1 & 1 & 1 \\
1 & 0 & 1 \\
1 & -\frac{c_{11} c_{36}-c_{16} c_{31}+c_{15} c_{36}-c_{16} c_{35}}{c_{13} c_{36}-c_{16} c_{33}} & -\frac{c_{11} c_{35}-c_{15} c_{31}-c_{15} c_{36}+c_{16} c_{35}}{\left(c_{13} c_{35}-c_{15} c_{33}\right.} \\
-\frac{c_{22}}{c_{24}} & 0 & -\frac{c_{22}}{c_{24}} \\
-\frac{c_{11} c_{36}-c_{16} c_{31}+c_{13} c_{36}-c_{16} c_{33}}{c_{15} c_{36}-c_{16} c_{35}} & 1 & \frac{c_{11} c_{33}-c_{13} c_{31}-c_{13} c_{36}+c_{16} c_{33}}{c_{13} c_{35}-c_{15} c_{33}} \\
\frac{c_{11} c_{35}-c_{15} c_{31}+c_{13} c_{35}-c_{15} c_{33}}{c_{15} c_{36}-c_{16} c_{35}} & \frac{c_{11} c_{33}-c_{13} c_{31}-c_{13} c_{35}+c_{15} c_{33}}{c_{13} c_{36}-c_{16} c_{33}} & 1
\end{array}\right]
$$

With the above found values for $n_{i j}(=0)$, with $i=1,2,3$ and $j=1,2,3$, and all $m_{i j}$ with $i=1, \ldots, 6$ and $j=1,2,3$, Eq. (74) reduces to

$$
\begin{gathered}
n_{14} m_{41}+n_{15} m_{51}+n_{16} m_{61}=1 \\
n_{15}+n_{16} m_{62}=0 \\
n_{14} m_{43}+n_{15} m_{53}+n_{16}=0 \\
n_{24} m_{41}+n_{25} m_{51}+n_{26} m_{61}=0 \\
n_{25}+n_{26} m_{62}=1 \\
n_{24} m_{43}+n_{25} m_{53}+n_{26}=0
\end{gathered}
$$

\section{5 of 25}




$$
\begin{gathered}
n_{34} m_{41}+n_{35} m_{51}+n_{36} m_{61}=0 \\
n_{35}+n_{36} m_{62}=0 \\
n_{34} m_{43}+n_{35} m_{53}+n_{36}=1
\end{gathered}
$$

where we have kept some of the $m_{i j}$-notation for the sake of clarity.

These equations can be solved by groups of three, e.g., Eqs. (97)-(99) are three equations with three unknowns $n_{14}, n_{15}$ and $n_{16}$. After some manipulation we find for the complete $\mathbf{N}$ :

$$
\mathbf{N}=\left[\begin{array}{cccc}
0 & 0 & 0 & -\frac{m_{53} * m_{62}-1}{m_{41}\left(1-m_{61}+m_{51} * m_{62}-m_{53} * m_{62}\right)} \\
0 & 0 & 0 & -\frac{m_{51}-m_{53} * m_{61}}{m_{41}\left(1-m_{61}+m_{51} * m_{62}-m_{53} * m_{62}\right)} \\
0 & 0 & 0 & -\frac{m_{61}-m_{51} * m_{62}}{m_{41}\left(1-m_{61}+m_{51} * m_{62}-m_{53} * m_{62}\right)}
\end{array}\right.
$$
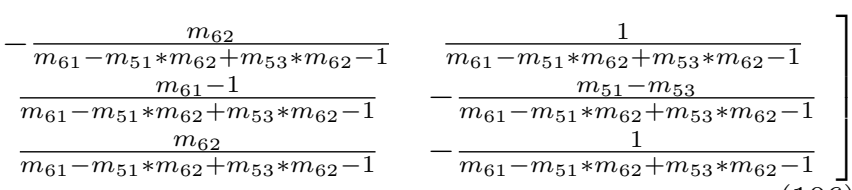

where, for obvious reasons, we have again kept some of the $m_{i j}$-notation. It is noted that both $\mathbf{M}$ and $\mathbf{N}$ are a function of the coefficients $c_{i j}$ (with $i=1,2,3$ and $j=1, \ldots, 6$ ). Because these coefficients are time varying - see Fig. 3 - both matrices are time varying.

Now that we have derived expressions for $\mathbf{M}$ and $\mathbf{N}$, we need to check Eq. (21) for stable zero dynamics:

$$
\begin{aligned}
\dot{\mathbf{z}}(t) & =\left(\dot{\mathbf{N}}\left(\mathbf{x}_{\mathbf{p}}, t\right)+\mathbf{N}\left(\mathbf{x}_{\mathbf{p}}, t\right) \mathbf{A}_{\mathbf{p}}\left(\mathbf{x}_{\mathbf{p}}, t\right)\right) \mathbf{M}\left(\mathbf{x}_{\mathbf{p}}, t\right) \mathbf{z}(t) \\
& =\mathbf{Z}_{\mathbf{p}}\left(\mathbf{x}_{\mathbf{p}}, t\right) \mathbf{z}(t)
\end{aligned}
$$

Evaluating for the complete trajectory the $3 \times 3$ matrix $\mathbf{Z}_{\mathbf{p}}$ at discrete time points, spaced by $\Delta t=0.5 \mathrm{~s}$ (where we have calculated $\dot{\mathbf{N}}$ by backward differencing) and calculating the eigenvalues gives us the plot of Fig. 6. The eigenvalues are a mix of a real one and a complex pair, and sometimes three real eigenvalues. They have in common that (mostly) the real parts are negative, hereby indicating stable zero dynamics. Only a few points show a positive real part. Inspecting the time history of the real parts, we find that this happens around $t=100 \mathrm{~s}$, but then only for a few seconds, see Fig. 7. Analyzing the individual matrices in Eq. (107) shows that the rapid change of $\mathbf{N}$ and thus large values for components of $\dot{\mathbf{N}}$ is the cause of this. Since both $\mathbf{M}$ and $\mathbf{N}$ are a function of $c_{i j}$, which are actually the gain values of a controller for the LTI system, this means that apparently the gains are changing rapidly. Going back to Fig. 3 confirms this: around $t=100 \mathrm{~s}$ the gains related to $\alpha$ and $\beta$ indeed change significanly. As explained earlier, this is caused by $\mathrm{a}(\mathrm{n})$ (sudden) increase in dynamic pressure, requiring larger thruster moments to counter possible large aerodynamic moments.

Since the gains are here only used to get an output vector (see Eq. (68)) and the system is not so sensitive to the particular values of the gains - note that the linear system with a constant gain matrix was still ASPR - it seems that by changing the rate of change of the gains this problem can be fixed. However, it may require a few iterations - we found that constant gains would not work for the non-linear system and therefore this is left as future work.

In conclusion, the current non-linear entry system fulfills the passivity conditions so the use of Simple Adaptive Control will lead to a stable controller configuration. It is expected that only a minor effort is required to correct the few $(\approx 3)$ seconds of flight, where the passivity conditions are not met.

\section{Example: Preliminary Control-System Design}

To conclude the paper the preliminary design of the attitude controller for the non-linear entry capsule is presented. For the nominal trajectory shown in Fig. 2 we will select two design points for which the weighting matrices $\mathbf{T}_{\mathbf{p}}$ and $\mathbf{T}_{\mathbf{i}}$ (Eqs. (5) and (6)) will be setup by means of a step-response analysis (Section V.A). Subsequently the controller performance is analyzed for the complete trajectory (Section V.B).

\section{A. Control System}

The general layout of the MRAC system is shown in Fig. 8. Five distinct elements can be identified, namely a Mission Management system providing, for instance, setpoints, the reference model, the adaptive-gain 


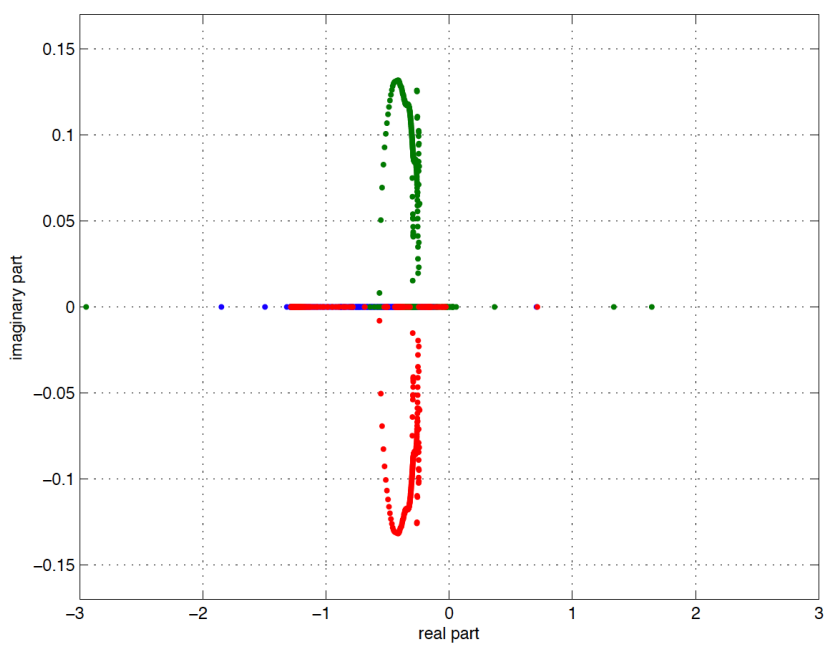

Figure 6. Eigenvalues for the zero-dynamics coefficient matrix $\mathbf{Z}_{\mathbf{p}}\left(\mathbf{x}_{\mathbf{p}}, t\right)$, evaluated at discrete time points for the nominal entry trajectory.

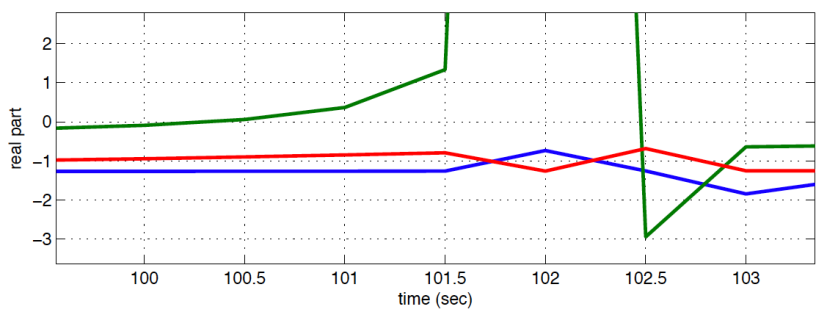

Figure 7. Real parts of the eigenvalues of the zero-dynamics coefficient matrix for a selected part of the flight. The maximum (positive) value is about 60, and concerns a single datapoint.

algorithm, the feed-forward compensators and the actual plant (Apollo). The reference model is a linearised state-space model of the entry capsule with decoupled longitudinal and lateral motion. For either motion channel, the system is stabilised by a state-feedback controller. The adaptive algorithm is based on Eqs. (1) through (9), and the corresponding weighting matrices will be determined during the design process. The feed-forward compensators around reference model and plant each may have the same basic form. They take the model control vector $\mathbf{u}_{\mathbf{m}}$ and plant control vector $\mathbf{u}_{\mathbf{p}}$ as input. However, in the current (preliminary) design we will not include them.

The three elements of the MRAC system, i.e., reference model, adaptive algorithm and compensators are all digitised using a zero-order hold discretisation scheme. The algebraic loops that are present in the loop for the feed-forward compensator as well as for the feed-back control system are removed by adding one-sample delays. The sample frequency for the controller has been put to $50 \mathrm{~Hz}$.

\section{Baseline design}

Usually, the approach to come to a converged design is the following. For a number of trajectory points, a state-space model is derived from the trimmed equilibrium state. For these linearised systems, an MRAC system is designed and the performance is optimised by either manually tuning the controller parameters or by applying an optimization technique. In case the weighting matrices $\mathbf{T}_{\mathbf{p}}$ and $\mathbf{T}_{\mathbf{i}}$ vary significantly due to the changing flight conditions, a(n) (linear) interpolation scheme between the related control-system designs (read: weighting matrices) should be applied.

Currently, we want to apply MRAC to a non-linear entry capsule that has to fly not only a nominal entry, but also cope with uncertainties. Therefore, as an example we will present the results for two design points using step responses. In the next Section V.B the performance for the complete trajectory will be 


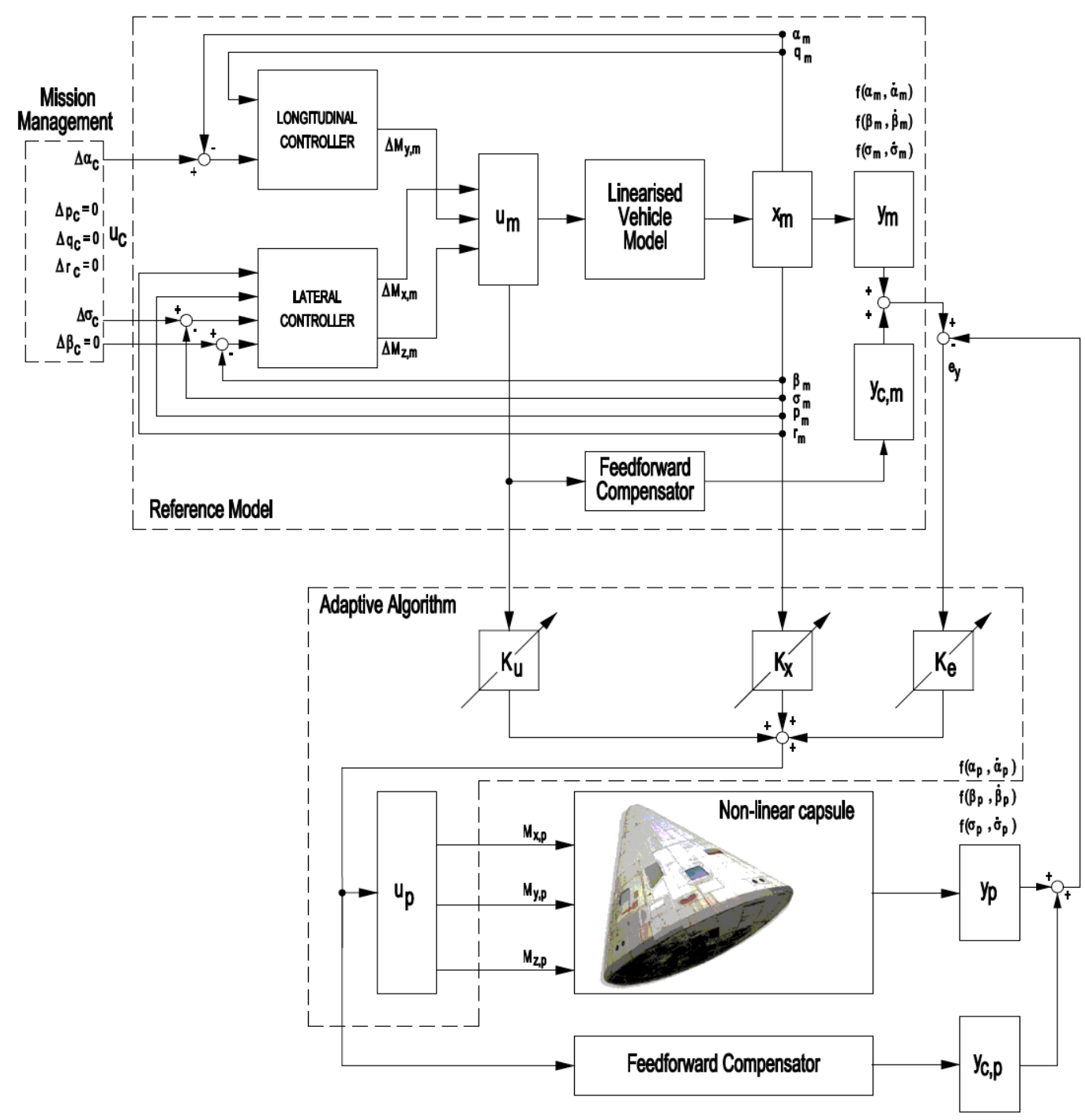

Figure 8. Model Reference Adapive Control implementation for the entry capsule.

discussed, as well as the controller robustness against uncertainties in mission parameters, vehicle design and environmental conditions.

Based on prior results, ${ }^{6}$ a linearised reference-model, stabilised by means of a linear state-feedback controller (LQR) was designed with maximum allowable state deviations of $\Delta \alpha_{\max }=1^{\circ}, \Delta \beta_{\max }=2^{\circ}, \Delta \sigma_{\max }=$ $3^{\circ}, \Delta p_{\max }=\Delta q_{\max }=\Delta r_{\max }=2^{\circ} / \mathrm{s}$. The maximum control effort is equal to the allowable ranges of the control effectuators, the reaction-control thrusters. For now, these have been chosen to be: $\Delta M_{T, x_{\max }}=500$ $\mathrm{Nm}$, and $\Delta M_{T, y_{\max }}=\Delta M_{T, z_{\max }}=1,000 \mathrm{Nm}$. The resulting reference-model control law is given by

$$
\mathbf{u}_{\mathbf{m}}=-\mathbf{K}_{\mathbf{m}}\left(\mathbf{x}_{c}-\mathbf{x}_{\mathbf{m}}\right)
$$

with $\mathbf{K}_{\mathbf{m}}$ being the LQR gain matrix and $\mathbf{x}_{\mathbf{c}}$ the commanded reference-model state, which follows from the command generator (or guidance system).

As mentioned, the damping of the system can be improved by including the error derivatives in the output error vector. The output errors for $\alpha, \beta$ and $\sigma$ become:

$$
e_{\alpha}=K_{\alpha}\left(\alpha_{m}-\alpha_{p}\right)+K_{\dot{\alpha}}\left(\dot{\alpha}_{m}-\dot{\alpha}_{p}\right)
$$




\begin{tabular}{c|cc|cc|cc}
\hline \hline & \multicolumn{2}{|c|}{ roll thrusters } & \multicolumn{2}{|c|}{ pitch thrusters } & \multicolumn{2}{c}{ yaw thrusters } \\
\hline element & $T_{p}$ & $T_{i}$ & $T_{p}$ & $T_{i}$ & $T_{p}$ & $T_{i}$ \\
\hline$e_{x}$ & 5 & 3 & - & - & - & - \\
$e_{y}$ & - & - & 80 & 24 & - & - \\
$e_{z}$ & - & - & - & - & 15 & 20 \\
$p_{m}$ & 10 & 3 & - & - & 30 & 30 \\
$q_{m}$ & - & - & 30 & 24 & - & - \\
$r_{m}$ & 4 & 3 & - & - & 30 & 30 \\
$\alpha_{m}$ & - & - & 30 & 24 & - & - \\
$\beta_{m}$ & 2 & 3 & - & - & 30 & 30 \\
$\sigma_{m}$ & 10 & 3 & - & - & 30 & 30 \\
$M_{T, x_{m}}$ & 1 & 3 & - & - & 7.5 & 5 \\
$M_{T, y_{m}}$ & - & - & 20 & 8 & - & - \\
$M_{T, z_{m}}$ & 0.3 & 0.5 & - & - & 30 & 10 \\
\hline \hline
\end{tabular}

Table 1. Weighting matrices for the low dynamic pressure design point

$$
\begin{aligned}
& e_{\beta}=K_{\beta}\left(\beta_{m}-\beta_{p}\right)+K_{\dot{\beta}}\left(\dot{\beta}_{m}-\dot{\beta}_{p}\right) \\
& e_{\sigma}=K_{\sigma}\left(\sigma_{m}-\sigma_{p}\right)+K_{\dot{\sigma}}\left(\dot{\sigma}_{m}-\dot{\sigma}_{p}\right)
\end{aligned}
$$

However, when there is a strong coupling between the three axes of motion, it is not possible to assign just a single error to a corresponding actuator, e.g., a yaw-thruster yields both a roll and a yaw moment due to the inertia coupling. This means that the errors should be blended together. A fairly simple way of blending would be to use the gain matrix $\mathbf{K}_{\mathbf{m}}$, like we did for the passivity analysis (Eq. (68)), since it is computed by using (linearised) information of the coupling between the axes (through $\mathbf{A}_{\mathbf{m}}$ and $\mathbf{B}_{\mathbf{m}}$ ). It means that we require full (rigid-body) state information about the plant, i.e.,

$$
\mathbf{y}_{\mathbf{p}}^{\mathbf{T}}=\mathbf{x}_{\mathbf{p}}^{\mathbf{T}}=\left(p_{p}, q_{p}, r_{p}, \alpha_{p}, \beta_{p}, \sigma_{p}\right)^{T}
$$

Note that in case of the presence of, for instance, elastic modes, one could still base the output error on the same rigid-body states, so full state knowledge would then not be required. Concluding, the output error becomes

$$
\mathbf{e}_{\mathbf{y}}=\mathbf{K}_{\mathbf{m}}\left(\mathbf{y}_{\mathbf{m}}-\mathbf{y}_{\mathbf{p}}\right)
$$

\section{Step Responses}

To design the control system, we select two design points and analyze the performance on the basis of step responses. The first point is chosen at the entry interface of $h=220 \mathrm{~km}$ and $V=11 \mathrm{~km} / \mathrm{s}$, and is characterized by a very low dynamic pressure. The atmosphere is very thin here, and for practical (control) purposes we can assume this to be part of the exoatmospheric mission phase. Note that due to the large velocity, the dynamic pressure will build up rapidly, resulting in large aerodynamic forces and moments. For a first design, only diagonal weighting matrices will be used. Each of the three thruster controls has its own set of matrices, which need to be tuned separately. The reference vector $\mathbf{r}$ in Eq. (1) has a dimension of 7 for roll and yaw control (the corresponding output error, i.e., $e_{y, x}$ or $e_{y, z}$, and $p_{m}, r_{m}, \beta_{m}, \sigma_{m}, M_{T, x_{m}}$ and $\left.M_{T, z_{m}}\right)$, and 4 for pitch control $\left(e_{y, x}, q_{m}, \alpha_{m}\right.$ and $\left.M_{T, y_{m}}\right)$. The applied (not optimised) weighting factors for the thrusters are listed in Table 1. Finally, the zero integral-gain values are chosen to be 0.1 for the error terms and 0.01 for the others. The $\sigma_{i}$ values are taken to be 0.1 , but only for the error terms.

As test for the controller performance, we define a simultaneous step function for the angle of attack $\left(\Delta \alpha_{\text {step }}=1^{\circ}\right)$ and bank angle $\left(\Delta \sigma_{\text {step }}=2^{\circ}\right)$, whereas for the angle of attack and angle of sideslip we assume errors in the initial condition of $\Delta \alpha_{0}=0.5^{\circ}$ and $\Delta \beta_{0}=1^{\circ}$. The initial angular rates are put to the trim 

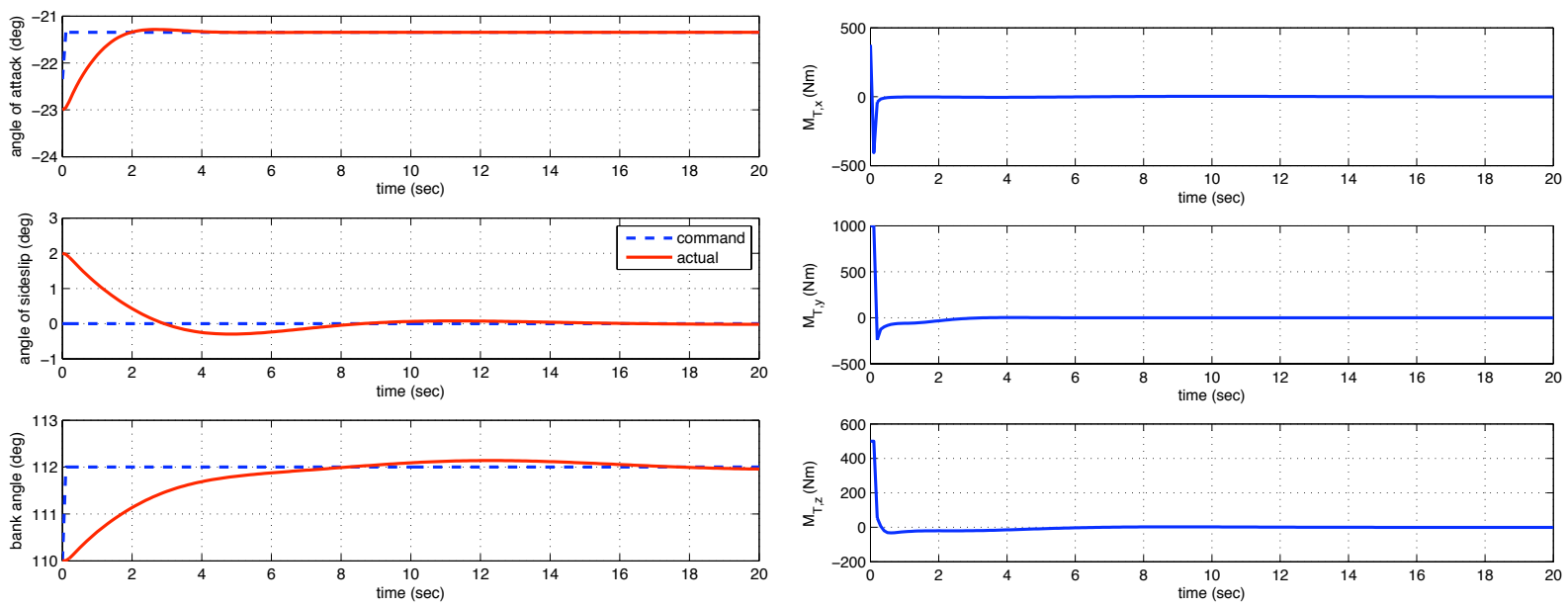

(a) Attitude-angle history.

(b) Thruster moments.

Figure 9. Low dynamic-pressure design point.

values that provide $\dot{\alpha}=\dot{\beta}=\dot{\sigma}=0$. The resulting attitude-angle response is shown in Fig. 9(a). It is obvious that the response is smooth and well-damped, without much overshoot. In Fig. 9(b) the corresponding thruster moments are plotted.

The second design point is one with a high dynamic pressure, i.e., around $\bar{q}=20,000 \mathrm{~N} / \mathrm{m}^{2}$, notably the conditions at $t=180 \mathrm{~s}$. The selected values for the weighting matrices are listed in Table 2 . As before, the zero integral-gain values are chosen to be 0.1 for the error terms and 0.01 for the others. The $\sigma_{i}$ values are taken to be 0.1, again only for the error terms. With a similar step function for the bank angle $\left(\Delta \sigma_{\text {step }}=2^{\circ}\right)$, and errors in the initial conditions of $\Delta \alpha_{0}=1^{\circ}$ and $\Delta \beta_{0}=1^{\circ}$, the response of the attitude angles is shown in Fig. 10(a). The response of the angle of attack has become very fast. This is due to the fact that the entry capsule is stable around its trim attitude, and the initial error forces the capsule back to this trim condition. The aerodynamic moment is in this case aiding the control. However, without control this motion would become oscillatory, albeit quite well damped. The control system improves the damping and suppresses the oscillatory motion. Note that applying a step function on the angle of attack would mean forcing the capsule away from its trim equilibrium. Since the aerodynamic moments will become large, even for small deviations from the trim attitude, it would require very large thruster moments to sustain this step. The current thrusters are by no means powerful enough. Finally, the small-amplitude angle-of-sideslip oscillation is a sensitivity in the control system that is normally filtered out, either by a filter on the error input channel, or by the finite step-accuracy (i.e., discretization) of the actuators. In Fig. 10(b), the corresponding thruster moments are shown.

\section{B. Results}

\section{Nominal Mission}

Using the weighting matrices that were established during the control-system design in the previous section, and using linear interpolation as a function of dynamic pressure, we will simulate now the complete trajectory (see Fig. 2 for the reference trajectory). From an uncontrolled entry simulation we see that despite the natural trim attitude, there appear high-frequency oscillations (Fig. 11) in case there are only small errors in the initial conditions. One of the objectives for the control system is to suppress these oscillations. Looking at the results of the controlled entry, i.e., the nominal mission, we see that indeed the oscillations are gone (Fig. 12(a) and Fig. 12(b)). It is clear that at some point $(t \approx 235 \mathrm{~s})$, when the capsule becomes less aerodynamically stable, the pitch thrusters saturate. However, the attitude motion remains very smooth. 


\begin{tabular}{c|cc|cc|cc}
\hline \hline & \multicolumn{2}{|c|}{ roll thrusters } & \multicolumn{2}{|c|}{ pitch thrusters } & \multicolumn{2}{c}{ yaw thrusters } \\
\hline element & $T_{p}$ & $T_{i}$ & $T_{p}$ & $T_{i}$ & $T_{p}$ & $T_{i}$ \\
\hline$e_{x}$ & 20 & 80 & - & - & - & - \\
$e_{y}$ & - & - & 5 & 2.5 & - & - \\
$e_{z}$ & - & - & - & - & 0.011 & 0.011 \\
$p_{m}$ & 40 & 20 & - & - & 0.55 & 0.55 \\
$q_{m}$ & - & - & 50 & 25 & - & - \\
$r_{m}$ & 40 & 40 & - & - & 0.11 & 0.11 \\
$\alpha_{m}$ & - & - & 100 & 25 & - & - \\
$\beta_{m}$ & 60 & 100 & - & - & 0.55 & 0.44 \\
$\sigma_{m}$ & 100 & 40 & - & - & 0.55 & 0.44 \\
$M_{T, x_{m}}$ & 20 & 20 & - & - & 0.022 & 0.022 \\
$M_{T, y_{m}}$ & - & - & 1 & 0.5 & - & - \\
$M_{T, z_{m}}$ & 10 & 10 & - & - & 0.022 & 0.022 \\
\hline \hline
\end{tabular}

Table 2. Weighting matrices for the high dynamic pressure design point

\section{Sensitivity Analysis}

For the (limited) sensitivity analysis we will consider two cases, i.e., a Monte Carlo analysis to get a picture of the overall performance and a single run with a selected set of maximum dispersions in the initial attitude and angular rates to get a feeling of the actual controller response. In all cases we run the simulations for $60 \mathrm{~s}$, which covers an altitude range of about $100 \mathrm{~km}$. When doing the Monte-Carlo runs, sampling the initial-condition errors from a uniform distribution with the attitude errors $\in[-5,5]^{\circ}$ and the angular-rate errors $\in[-5,5]^{\circ} / \mathrm{s}$, it appeared that there are quite some combinations of errors that resulted in controls oscillating between the minimum and maximum values. So, for these errors the weighting matrices need to be re-tuned, possibly through an optimization process. This remains to be done as future work. To show some results we will reduce the maximum initial-condition errors to $[-3,3]^{\circ}$ and $[-1,1]^{\circ} / \mathrm{s}$, and will also include a $-5 \%$ to $5 \%$ error on the inertia properties, $I_{x x}, I_{y y}, I_{z z}$ and $I_{x z}$. A total of 200 runs will be executed.

To get an indication of the performance we define 6 performance metrices:

$$
\begin{gathered}
\sum_{\alpha_{\text {err }}}=\int_{0}^{t} \sqrt{\left(\alpha_{c}-\alpha_{p}\right)^{2}} d t \quad \sum_{\beta_{\text {err }}}=\int_{0}^{t} \sqrt{\left(\beta_{c}-\beta_{p}\right)^{2}} d t \quad \sum_{\sigma_{\text {err }}}=\int_{0}^{t} \sqrt{\left(\sigma_{c}-\sigma_{p}\right)^{2}} d t \\
\sum_{x}=\int_{0}^{t}\left|M_{T, x}\right| d t \quad \sum_{y}=\int_{0}^{t}\left|M_{T, y}\right| d t \quad \sum_{z}=\int_{0}^{t}\left|M_{T, z}\right| d t
\end{gathered}
$$

These metrices are the integrated state deviation (attitude angles) and the integrated control effort. It is obvious that these metrices should all be as small as possible.

To begin with the single run, we have defined $\Delta \alpha_{0}=3^{\circ}, \Delta \beta_{0}=-3^{\circ}, \Delta \sigma_{0}=3^{\circ}, \Delta p=-1^{\circ} / \mathrm{s}, \Delta q=-1^{\circ} / \mathrm{s}$ and $\Delta r=1^{\circ} / \mathrm{s}$. The corresponding responses and thruster moments are shown in Fig. 13(a) and Fig. 13(b), respectively. The responses are smooth, and well within the range of the RCS thrusters. For reference, the shown responses give numerical values of these metrices of $\sum_{\alpha_{\text {err }}}=3.57^{\circ} \mathrm{s}, \sum_{\beta_{\text {err }}}=7.70^{\circ} \mathrm{s}, \sum_{\sigma_{\text {err }}}=7.52^{\circ} \mathrm{s}$, $\sum_{x}=146.2 \mathrm{Nms}, \sum_{y}=488.3 \mathrm{Nms}$ and $\sum_{z}=341.1 \mathrm{Nms}$. Note that although the curves are shown for the interval 0-20 s, the metrices have been calculated for the full $60 \mathrm{~s}$ of simulation.

Finally, the results of the Monte-Carlo runs are shown in Fig. 14. From the results it can be concluded that the metrices are in the same order of magnitude as those for the single run (in fact, the mean value is somewhat smaller). This leads to believe that the individual responses will be similar, which is an acceptable result. Of course, more simulations need to be done as well as a more detailed analysis of the results. This remains to be done as future work. 

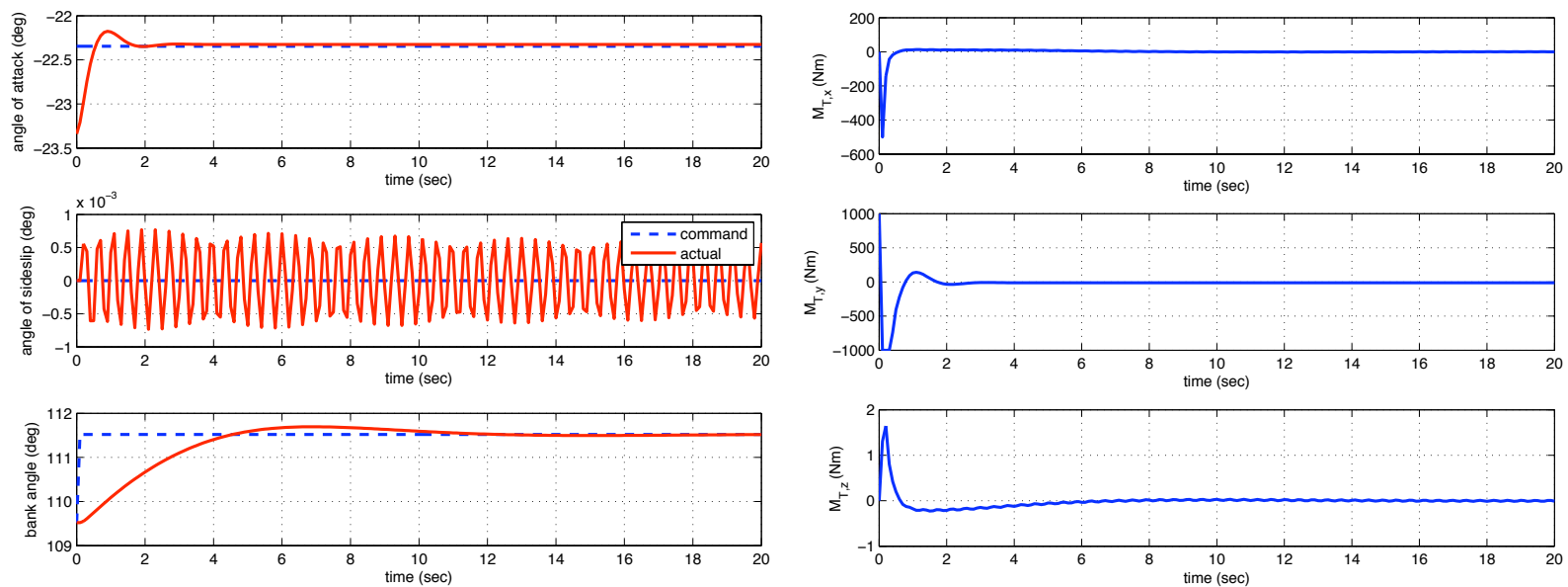

(a) Attitude-angle history.

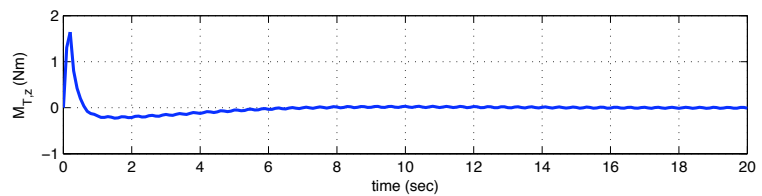

(b) Thruster moments.

Figure 10. High dynamic-pressure design point.
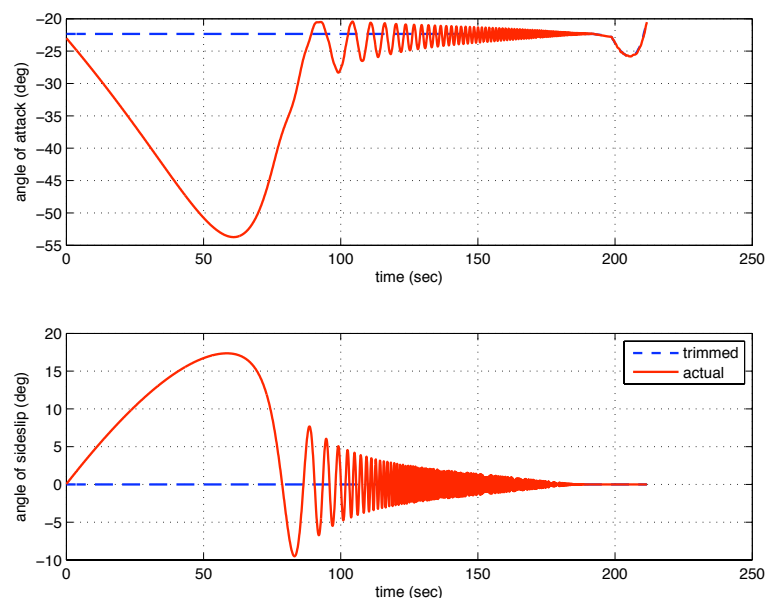

Figure 11. Attitude-angle history for an uncontrolled entry, with errors in the initial pitch and yaw rate of $0.25^{\circ} / \mathrm{s}$.

\section{Conclusions and Recommendations}

In this paper, the stability properties for a control-system design based on Simple Adaptive Control theory are studied for a non-linear and non-stationary entry capsule. Even though previous implementations for winged re-entry vehicles have been successful without theoretical stability analysis, an effort was made to obtain the analytical expressions that would allow the test of the sufficient passivity conditions that guarantee the robustness of system stability. The conclusion of this work is that the ASP conditions are satisfied for the non-linear system. As these are sufficient conditions, one can therefore safely use the adaptive controller.

Subsequently, the preliminary design of a control system for the entry capsule using reaction-control thrusters around each of the three axes is discussed. The two designs for both a low and a high dynamicpressure operating condition show an excellent performance. Each design uses its own controller parameters (i.e., weighting matrices, zero integral gain and integral-gain filter parameter), which necessitates the use of an interpolation scheme once a trajectory with changing operating conditions is flown.

The nominal mission (entry at $220 \mathrm{~km}$ altitude with a velocity of $11 \mathrm{~km} / \mathrm{s}$, and final conditions at Mach $=1$ ) can be flown without any difficulty. The angle-of-attack command is provided by a trim law, and although some deviations are observed when the capsule becomes less aerodynamically stable, this deviation 

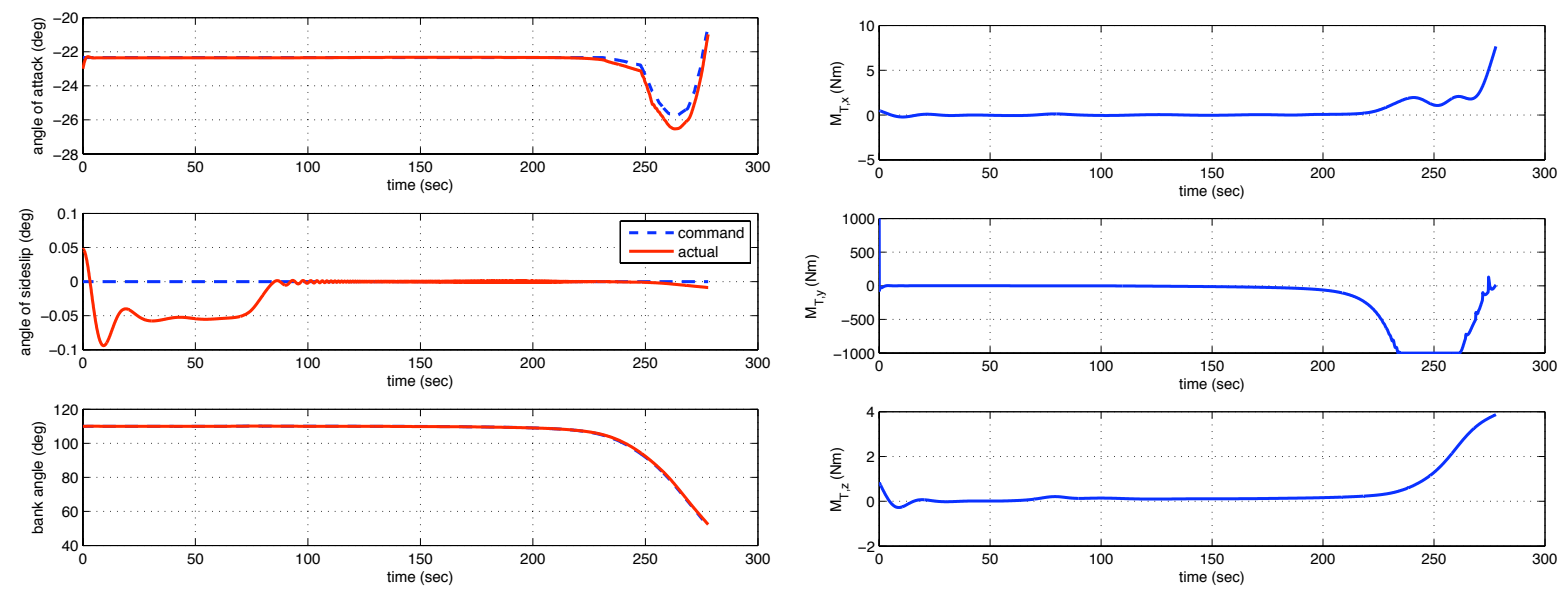

(a) Attitude-angle history.

(b) Thruster moments.

Figure 12. Nominal-mission results.

does not diverge in spite of the pitch-thruster saturation. The response due to errors in the initial attitude and angular rate shows smooth curves in both attitude motion and thruster controls. A Monte-Carlo analysis that includes errors in the initial attitude, angular rate and inertia properties shows similar results, and it can be concluded that the performance of the controller given the current dispersions is satisfying.

Due to the changing gains when the dynamic pressure starts building up, there is a brief period of about 3 seconds where the passivity conditions are not met, because these gains are used in the output definition of the entry capsule. By changing the rate of change of the gains this problem can be fixed, although it may require a few (analysis) iterations. This will have to be the first step in the (on-going) research about stability of non-linear entry systems. Concerning the control-system design, future work aims at fine tuning the controller parameters, possibly through some optimization process, and to include more error sources in the sensitivity analysis. The design of a guidance system, finally, will enable a complete analysis of a guidance and control system for re-entry-capsules.

\section{Acknowledgments}

I would like to thank Itzhak Barkana for his insight and the many discussions that we have had about Simple Adaptive Control in general, and about stability of non-linear systems in particular.

\section{References}

\footnotetext{
${ }^{1}$ Barkana, I., "Classical and Simple Adaptive Control for Non-Minimum Phase Autopilot Design", Journal of Guidance, Control, and Dynamics, Vol. 24, No. 4, pp. 631-638, July-August 2004.

${ }^{2}$ Kaufman, H., Barkana, I. and Sobel, K., Direct adaptive control algorithms: Theory and applications, Second edition, Springer-Verlag, New York, 1998.

${ }^{3}$ Barkana, I., Output Feedback Stabilizability and Passivity in Nonstationary and Nonlinear Systems, International Journal of Adaptive Control and Signal Processing, Vol. 24, No. 7, pp. 568-591, July 2010 (Published on-line 3 November 2009).

${ }^{4}$ Mehiel, E.A. and Balas, M.J., "Adaptive Control for a Deployable Optical Telescope", AIAA-04-5222, From: AIAA Guidance, Navigation, and Control Conference, Providence, RI, August 16-19, 2004.

${ }^{5}$ Mooij, E., "Model Reference Adaptive Guidance for Re-entry Trajectory Tracking ", AIAA-04-4775, From: AIAA Guidance, Navigation, and Control Conference, Providence, RI, August 16-19, 2004a.

${ }^{6}$ Mooij, E., "Simple Adaptive Bank-Reversal Control of a winged Re-entry Vehicle ", AIAA-04-4869, From: AIAA Guidance, Navigation, and Control Conference, Providence, RI, August 16-19, 2004b.

${ }^{7}$ Mooij, E., Robustness Analysis of an Adaptive Re-entry Guidance System", AIAA-05-6146, AIAA Guidance, Navigation, and Control Conference, San Francisco, CA, August 15-18, 2005.

${ }^{8}$ Mooij, E. and Barkana, I., "Theoretical Stability Analysis of Simple Adaptive Control for a Winged Re-entry Vehicle", AIAA-06-6417, From: AIAA Guidance, Navigation, and Control Conference, August 21-24, 2006, Keystone, CO.

${ }^{9}$ Barkana, I., "Comment on Design of Strictly Positive Real Systems Using Constant Output Feedback", IEEE Transactions on Automatic Control, Vol. 49, No. 11, November 2004.
} 

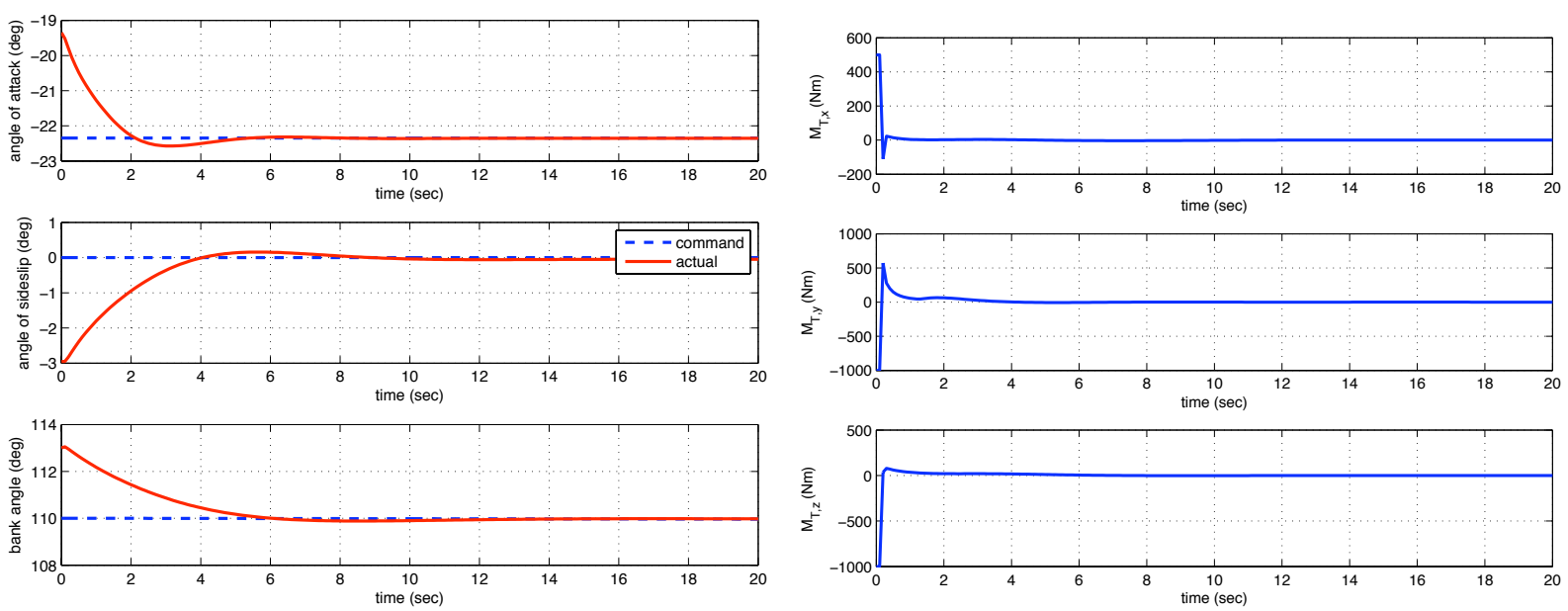

(a) Attitude-angle history.

(b) Thruster moments.

Figure 13. Combined errors in the initial conditions, single run.
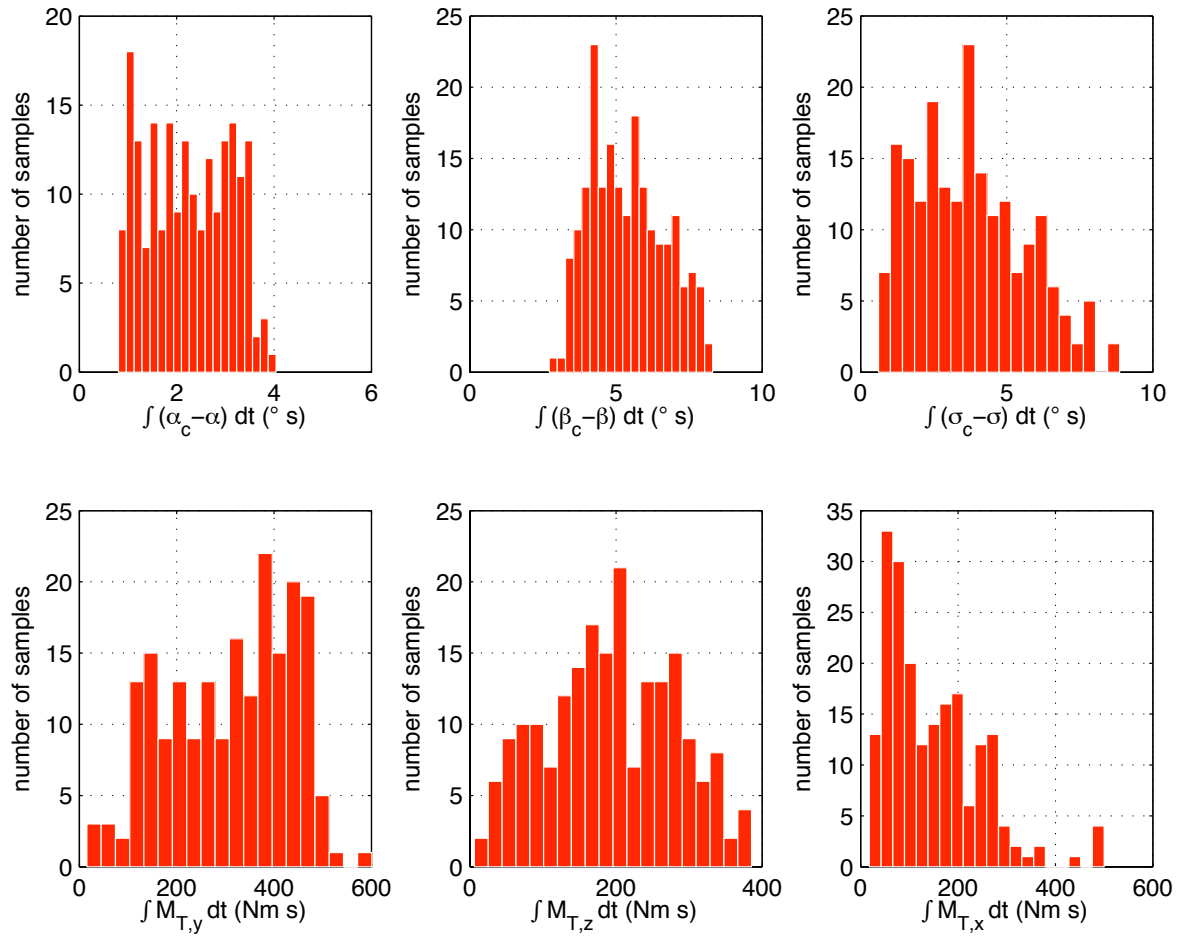

Figure 14. Performance metrices for the 200 Monte-Carlo runs. 
${ }^{10}$ Barkana, I. and Kaufman, H., "Global Stability and Performance of an Adaptive Control Algorithm", International Journal of Control, 46(6), pp. 1491-1505, 1985.

${ }^{11} \mathrm{Hsu}$, L., and R. Costa, "MIMO direct adaptive control with reduced prior knowledge of the high-frequency gain", Proceedings of $38^{t} h$ IEEE Conference on Decision and Control, Phoenix, AZ, pp. 3303-3308, 1999.

${ }^{12}$ Mooij, E. "Passivity Analysis for Non-Linear, Non-Stationary Entry Capsules: Translational Motion", From: IFAC 18 $8^{t h}$ World Congress, August 28-September 2, 2011, Milan, Italy.

${ }^{13}$ Vinh, N.X., Optimal trajectories in atmospheric flight, Elsevier scientific publishing company, 1981.

${ }^{14}$ Mooij, E., "Aerospace-Plane Flight Dynamics. Analysis of Guidance and Control Concepts", Ph.D. dissertation, Delft University of Technology, 1998. Available from http://repository.tudelft.nl/

${ }^{15}$ North American Aviation, Inc., "Aerodynamic Data Manual for Project Apollo", NASA-CR-82907, 1 January 1965.

${ }^{16}$ Moseley, Jr., W.C., Moore Jr., R.H. and Hughes, J.E., "Stability Characteristics of the Apollo Command Module", NASA TN D-3890, March 1967. 